\title{
On the First-Order Shape Derivative of the Kohn-Vogelius Cost Functional of the Bernoulli Problem
}

\author{
Jerico B. Bacani ${ }^{1,2}$ and Gunther Peichl ${ }^{2}$ \\ ${ }^{1}$ Department of Mathematics and Computer Science, University of the Philippines Baguio, Governor Pack Road, \\ Baguio City 2600, Philippines \\ ${ }^{2}$ Institute for Mathematics and Scientific Computing, University of Graz, Heinrichstrasse 36, A-8010 Graz, Austria \\ Correspondence should be addressed to Jerico B. Bacani; jicderivative@yahoo.com
}

Received 18 August 2013; Accepted 1 November 2013

Academic Editor: Sergei V. Pereverzyev

Copyright (C) 2013 J. B. Bacani and G. Peichl. This is an open access article distributed under the Creative Commons Attribution License, which permits unrestricted use, distribution, and reproduction in any medium, provided the original work is properly cited.

\begin{abstract}
The exterior Bernoulli free boundary problem is being considered. The solution to the problem is studied via shape optimization techniques. The goal is to determine a domain having a specific regularity that gives a minimum value for the Kohn-Vogeliustype cost functional while simultaneously solving two PDE constraints: a pure Dirichlet boundary value problem and a Neumann boundary value problem. This paper focuses on the rigorous computation of the first-order shape derivative of the cost functional using the Hölder continuity of the state variables and not the usual approach which uses the shape derivatives of states.
\end{abstract}

\section{Introduction}

The Bernoulli problem is the prototype of a stationary free boundary problem. It arises in various applications such as electrochemical machining, potential flow in fluid mechanics, tumor growth, optimal insulation, molecular diffusion, and steel and glass production [1-6]. A characteristic feature of free boundary problems is that not only the state variable is unknown but also the domain on which the state equation is posed. This represents a significant theoretical as well as numerical challenge. One can characterize the Bernoulli problem, at least along general lines, by finding a connected domain as well as a function which is harmonic on this domain. One component on the boundary is known. The other one is determined by a set of overdetermined boundary conditions (a Dirichlet condition and a Neumann condition) for the state. If the free boundary component is strictly exterior to the fixed part of the boundary, the problem is called exterior Bernoulli problem and interior Bernoulli problem otherwise. For more discussions related to interior and exterior Bernoulli problems, we refer the reader to $[1,4$, 7-10].

Recent strategies to compute a numerical solution are based on reformulating the Bernoulli problem as a shape optimization problem. This can be achieved in several ways. For a given domain, one can choose one of the boundary conditions on the free boundary to obtain a well-posed state equation. The domain is determined by the requirement that the other condition on the free boundary is satisfied in a least squares sense (cf. [11-13]). Alternatively, one can compute on a given domain two auxiliary states: $u_{D}$ which satisfies the Dirichlet condition and $u_{N}$ which satisfies the Neumann condition on the free boundary. The underlying domain is selected such that the difference $J(\Omega)=\left|u_{D}-u_{N}\right|_{H^{1}(\Omega)}^{2}$ is as small as possible. In fact, if $J(\Omega)=0$ for a domain $\Omega$ then $u_{D}=u_{N}$ and $\left(u_{D}, \Omega\right)$ is a solution of the Bernoulli problem. Sometimes $J$ is called Kohn-Vogelius functional since Kohn and Vogelius were among the first who used such a functional in the context of inverse problems [14]. Standard algorithms to minimize $J$ require some gradient information. So in this paper, the first-order sensitivity analysis is carried out for the functional $J$ for the exterior Bernoulli problem. The main contribution in this paper is the application of a shape optimization technique that leads to the explicit expression for the shape derivative of the cost functional. This is done through variational means similar to the techniques developed in $[9,10,13]$, wherein we use the Hölder continuity of the state variables satisfying the Dirichlet and Neumann 
problems but we do not introduce any adjoint variables. In our approach, we also bypass the use of the material derivatives of the states (which was done in [1]) and the use of states' shape derivatives.

The rest of the paper is structured as follows. Section 2 presents the Bernoulli free boundary problem and its shape optimization formulations. Section 3 provides a list of shape optimization tools that are needed in the analysis for the shape derivatives of the Kohn-Vogelius cost functional $J$. Section 4 presents an exhaustive discussion on the first-order shape derivative of $J$. Finally, Section 5 draws conclusion and observation.

\section{The Bernoulli Problem}

The exterior Bernoulli free boundary problem is formulated as follows. Given a bounded and connected domain $A \subset \mathbb{R}^{2}$ with a fixed boundary $\Gamma:=\partial A$ and a constant $\lambda<0$, one needs to find a bounded connected domain $B \subset \mathbb{R}^{2}$ with a free boundary $\Sigma$, containing the closure of $A$, and an associated state function $u: \Omega \rightarrow \mathbb{R}$, where $\Omega=B \backslash \bar{A}$, such that the overdetermined conditions are satisfied:

$$
\begin{array}{cc}
-\Delta u=0 & \text { in } \Omega, \\
u=1 & \text { on } \Gamma, \\
u=0 & \text { on } \Sigma, \\
\frac{\partial u}{\partial \mathbf{n}}=\lambda & \text { on } \Sigma .
\end{array}
$$

On the other hand, the interior Bernoulli free boundary problem has the following formulation. Given a bounded and connected domain $B \subset \mathbb{R}^{2}$ with a fixed boundary $\Gamma:=\partial B$ and a constant $\lambda>0$, one determines a bounded connected domain $A \subset \bar{B}$ with a free boundary $\Sigma$ and an associated state function $u: \Omega \rightarrow \mathbb{R}$, where $\Omega=B \backslash \bar{A}$, subject to the following constraints:

$$
\begin{gathered}
-\Delta u=0 \quad \text { in } \Omega, \\
u=0 \quad \text { on } \Gamma, \\
u=1 \quad \text { on } \Sigma, \\
\frac{\partial u}{\partial \mathbf{n}}=\lambda \quad \text { on } \Sigma .
\end{gathered}
$$

In both problems $\mathbf{n}$ is the outward unit normal vector to $\Sigma$. The difference in the domains of these two types of Bernoulli problems is depicted in Figure 1.

Methods of shape optimization can be employed in solving the exterior Bernoulli free boundary problem (1). As we observe, this boundary problem is ill-posed due to the fact that we have overdetermined conditions on the free boundary $\Sigma$. So to overcome the difficulty of solving it, one can reformulate it as one of the following shape optimization problems which involves now a well-posed state equation.

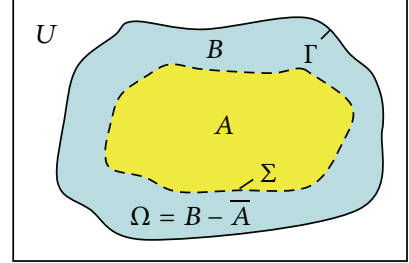

(a)

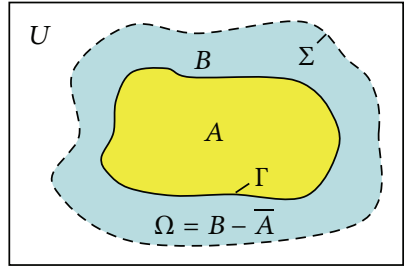

(b)
FIGURE 1: The domain $\Omega$ for the interior Bernoulli problem (a) and exterior problem (b).

(1) Tracking Neumann data $[11,12]$ as

$$
\min _{\Sigma} J_{1}(\Sigma) \equiv \min _{\Sigma} \frac{1}{2} \int_{\Sigma}\left(\frac{\partial u_{D}}{\partial \mathbf{n}}-\lambda\right)^{2} d s
$$

where the state function $u_{D}$ is the solution to the Dirichlet problem

$$
\begin{array}{cc}
-\Delta u_{D}=0 & \text { in } \Omega, \\
u_{D}=1 & \text { on } \Gamma, \\
u_{D}=0 & \text { on } \Sigma .
\end{array}
$$

(2) Tracking Dirichlet data $[11,13]$ as

$$
\min _{\Sigma} J_{2}(\Sigma) \equiv \min _{\Sigma} \frac{1}{2} \int_{\Sigma} u_{N}^{2} d s
$$

where the state function $u_{N}$ is the solution to the Neumann problem

$$
\begin{gathered}
-\Delta u_{N}=0 \quad \text { in } \Omega, \\
u_{N}=1 \quad \text { on } \Gamma, \\
\frac{\partial u_{N}}{\partial \mathbf{n}}=\lambda \quad \text { on } \Sigma .
\end{gathered}
$$

(3) Minimizing the Kohn-Vogelius type cost functional $[12,15]$ as

$$
\min _{\Omega} J(\Omega) \equiv \min _{\Omega} \frac{1}{2} \int_{\Omega}\left|\nabla\left(u_{D}-u_{N}\right)\right|^{2} d x,
$$

where state functions $u_{D}$ and $u_{N}$ satisfy (4) and (6), respectively.

In this paper, we are just interested in the study of minimizing the Kohn-Vogelius functional $J$.

\section{Tools in Shape Optimization}

3.1. Feasible Domain $\Omega$. In this work, we are interested in $C^{k, 1}$-domains, where $k \geq 0$. Aside from being $C^{k, 1}$ we also assume that these are bounded and connected subsets of a bigger set $U$ which is also a bounded connected $C^{k, 1}$ domain. This $U$ is called the universal or the hold-all domain. The 
smoothness of these domains can be defined in the following sense (cf. [16]).

Consider the standard unit orthonormal basis $\left\{e_{1}, e_{2}, \ldots\right.$, $\left.e_{n}\right\}$ in $\mathbb{R}^{n}$. For a point $x=\left(x_{1}, x_{2}, \ldots, x_{n}\right) \in \mathbb{R}^{n}$, let $x^{\prime}=$ $\left(x_{1}, x_{2}, \ldots, x_{n-1}\right) \in \mathbb{R}^{n-1}$ so as to write $x=\left(x^{\prime}, x_{n}\right)$. Consider the unit ball $B(0,1)$ and introduce the subsets

$$
\begin{aligned}
B^{+}(0,1) & :=\left\{x \in B(0,1): x_{n}>0\right\}, \\
B^{-}(0,1) & :=\left\{x \in B(0,1): x_{n}<0\right\}, \\
B^{0}(0,1) & :=\left\{x \in B(0,1): x_{n}=0\right\} .
\end{aligned}
$$

Definition 1. A domain $\Omega \subset \mathbb{R}^{n}$ with a nonempty boundary $\partial \Omega$ is called a $C^{k, l}$-domain, where $0 \leq k, 0<l \leq 1$, if for every $y \in \partial \Omega$ there exists a neighborhood $N_{y}$ of $y$ and a $C^{k, l}$ diffeomorphism $f_{y}: N_{y} \rightarrow B(0,1)$ such that (a) $f_{y}\left(N_{y} \cap\right.$ $\Omega)=B^{+}(0,1)$, (b) $f_{y}\left(N_{y} \cap \partial \Omega\right)=B^{0}(0,1)$, and (c) $f_{y}\left(N_{y} \cap\right.$ $\left.\bar{\Omega}^{c}\right)=B^{-}(0,1)$.

To illustrate this for $n=2$ and $k=l=1$, see Figure 2 .

Note that if $\Omega$ is a bounded, open, connected set with a $C^{0,1}$ boundary, then int $\bar{\Omega}=\Omega$. This was given in [17] and we prove it as follows.

Theorem 2 (see [17]). If $\Omega$ is a bounded open connected subset of $\mathbb{R}^{n}$ with Lipschitz continuous boundary, then int $\bar{\Omega}=\Omega$.

Proof. The interior of $\bar{\Omega}$ is the largest open set contained in the set $\bar{\Omega}$. Moreover, $\Omega \subseteq \bar{\Omega}$. It follows that $\Omega \subseteq$ int $\bar{\Omega}$. Next, we show that int $\bar{\Omega} \subseteq \Omega$. Clearly, int $\bar{\Omega} \subseteq \bar{\Omega}$. We now show that if $x \in$ int $\bar{\Omega}$, then $x \notin \partial \Omega$.

Suppose $x \in \partial \Omega$ and $x \in$ int $\bar{\Omega}$. We need to show that any open set $N$ containing $x$ contains an element not in $\bar{\Omega}$. We first note that by definition of $C^{0,1}$ domain, there exists a neighborhood $N_{x}$ of $x \in \partial \Omega$ and a diffeomorphism $f_{x}$ : $N_{x} \rightarrow B(0,1)$. Let $N$ be an open set containing $x$ with $N \subset$ $N_{x}$. It follows that $f_{x}(N)$ is an open set containing 0 and this set is contained in $B(0,1)$. Hence, there exists $z \in f_{x}(N)$ such that $z \in B^{-}(0,1)$. This implies that $f_{x}^{-1}(z) \in N \cap \bar{\Omega}^{c}$. Thus, $N$ contains an element not in $\bar{\Omega}$, which is a contradiction. Therefore, $x \notin$ int $\bar{\Omega}$. We have proven that if $x \in \partial \Omega$, then $x \notin$ int $\bar{\Omega}$. Taking the contrapositive of this statement we get that if $x \in$ int $\bar{\Omega}$, then $x \notin \partial \Omega$. Since $x \in \bar{\Omega}$ but $x \notin \partial \Omega$, we conclude $x \in \Omega$. Thus, int $\bar{\Omega} \subseteq \Omega$. We have shown that $\Omega \subseteq$ int $\bar{\Omega}$ and int $\bar{\Omega} \subseteq \Omega$. Therefore, int $\bar{\Omega}=\Omega$.

3.2. The Perturbation of Identity Technique. Given bounded connected domains $\Omega$ and $U$ of $\mathbb{R}^{2}$, where $\Omega \subseteq U$, and a linear space $\Theta$ of vector fields $\mathbf{V}$, one can deform $\Omega$ via the perturbation of identity operator

$$
T_{t}: \bar{U} \longrightarrow \mathbb{R}^{2}, \quad T_{t}(x)=x+t \mathbf{V}(x), \quad x \in \bar{U},
$$

where $\mathbf{V} \in \Theta$. For a given $t$ we denote the deformed domain to be $\Omega_{t}$, which is the image of $\Omega$ under $T_{t}$.

Throughout the paper, we use the usual infinity norms in the spaces $C(X ; \mathbb{R}), C\left(X ; \mathbb{R}^{2}\right)$, and $C\left(X ; \mathbb{R}^{2 \times 2}\right)$, where $X$ is a

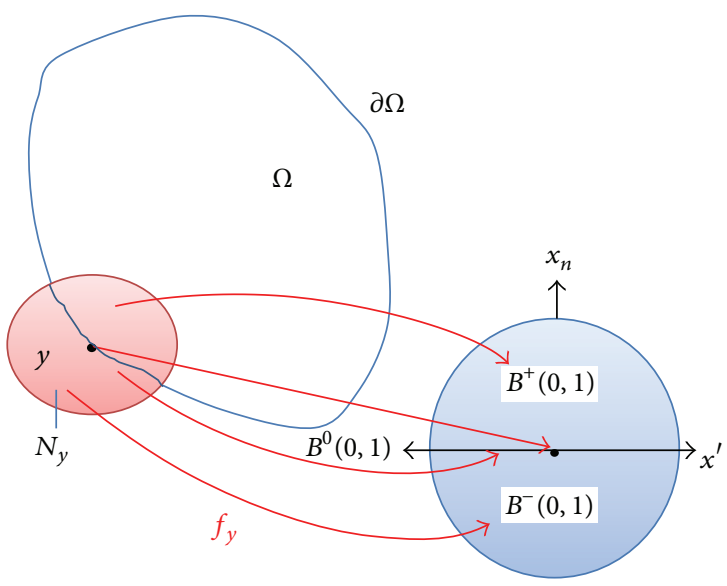

FIGURE 2: A $C^{k, 1}$-domain $\Omega$, where $f_{y}$ is a diffeomorphism from the neighborhood $N_{y}$ to the ball $B(0,1)$.

compact subset of $\mathbb{R}^{2}$. In addition to this, we also denote the Frobenius norm of $M(x)$ to be

$$
|M(x)|_{F}=\left(\sum_{i, j=1}^{2}\left|M_{i j}(x)\right|^{2}\right)^{1 / 2} \text {. }
$$

This norm and the infinity norm of the matrix $M$ can be related as

$$
|M(x)|_{F} \leq 2|M|_{\infty}, \quad x \in X .
$$

This can be shown easily. One can also show that if $M \epsilon$ $C\left(X ; \mathbb{R}^{2 \times 2}\right)$ and $y \in L^{2}\left(X ; \mathbb{R}^{2}\right)$, then the vector $M y$ is bounded in $L^{2}\left(X ; \mathbb{R}^{2}\right)$. In fact,

$$
|M y|_{L^{2}(X)} \leq 2|M|_{\infty}|y|_{L^{2}(X)}
$$

and the proof is trivial. Finally, the symbols $|\cdot|$ or $|\cdot|_{2}$ will refer to the usual Euclidean norm.

The Perturbed Domain $\Omega_{t}$. The domains $\Omega_{t}$ that are considered in this work are of annulus type with boundary $\partial \Omega_{t}$, which is the union of two disjoint sets $\Gamma_{t}$ and $\Sigma_{t}$, referred to as the fixed and free boundaries, respectively. These domains are obtained through the operator defined in (9), where V belongs to $\Theta$, which is defined as

$$
\Theta=\left\{\mathbf{V} \in C^{1,1}\left(\bar{U}, \mathbb{R}^{2}\right):\left.\mathbf{V}\right|_{\Gamma \cup \partial U}=0\right\} .
$$

For $t=0$, we obtain the reference domain $\Omega:=\Omega_{0}$, with a fixed boundary $\Gamma:=\Gamma_{0}$ and a free boundary $\Sigma:=\Sigma_{0}$.

The main objective in this subsection is to show that $T_{t}$ is a diffeomorphism from $\Omega$ to $\Omega_{t}$ for sufficiently small $t$. To verify this, we need the following results, which are given and proven in [17].

Theorem 3. If $\Omega$ is a bounded, open, connected set in $\mathbb{R}^{n}$ such that int $\bar{\Omega}=\Omega$ and $\varphi$ is a continuous injective mapping from $\bar{\Omega}$ to $\mathbb{R}^{n}$, then

$$
\begin{gathered}
\varphi(\bar{\Omega})=\overline{\varphi(\Omega)}, \quad \varphi(\Omega)=\operatorname{int} \varphi(\bar{\Omega}), \\
\varphi(\partial \Omega)=\partial \varphi(\Omega)=\partial \varphi(\bar{\Omega}) .
\end{gathered}
$$




\section{Theorem 4. Suppose}

(1) $\Omega$ is a bounded, open, connected set in $\mathbb{R}^{n}$ such that int $\bar{\Omega}=\Omega$,

(2) $\varphi_{0} \in C\left(\bar{\Omega}, \mathbb{R}^{2}\right)$ where $\varphi_{0}$ is injective,

(3) $\varphi \in C\left(\bar{\Omega}, \mathbb{R}^{2}\right) \cap C^{1}\left(\Omega, \mathbb{R}^{2}\right)$ such that

$$
\begin{aligned}
& \operatorname{det} D \varphi(x)>0 \quad \forall x \in \Omega, \\
& \varphi(x)=\varphi_{0}(x) \quad \forall x \in \partial \Omega .
\end{aligned}
$$

Then

(i) $\varphi: \bar{\Omega} \rightarrow \varphi(\bar{\Omega})$ is a homeomorphism (i.e., $\varphi$ is a bijection, $\varphi$ is continuous, and $\varphi^{-1}$ is continuous),

(ii) $\varphi: \Omega \rightarrow \varphi(\Omega)$ is a $C^{1}$-diffeomorphism (i.e., $\varphi$ is a bijection, $\left.\varphi \in C^{1}(\Omega), \varphi^{-1} \in C^{1}(\varphi(\Omega))\right)$,

(iii) $\varphi(\Omega)=\varphi_{0}(\Omega), \varphi(\bar{\Omega})=\varphi_{0}(\bar{\Omega})$.

We also consider the following property of a domain, which is also found in [17, page 52].

Lemma 5. If $\Omega$ is a bounded, open, connected subset of $\mathbb{R}^{n}$ having a Lipschitz continuous boundary, then there is a number $c(\Omega)$ such that, for any given points $x, y \in \bar{\Omega}$, one can find a finite sequence of points $y_{k}, k=1, \ldots, l+1$, satisfying the following properties:

(a) $y_{1}=x, y_{k} \in \Omega$ for $2 \leq k \leq l, y_{l+1}=y$,

(b) $\left(y_{k}, y_{k+1}\right) \subset \Omega$ for $1 \leq k \leq l$,

(c) $\sum_{k=1}^{l}\left|y_{k+1}-y_{k}\right| \leq c(\Omega)|x-y|$.

We also recall the useful property of the determinant of the Jacobian of $T_{t}$ which is given in the next lemma. Here we use the notation

$$
I_{t}(x)=\operatorname{det} D T_{t}(x), \quad x \in \bar{U}
$$

Lemma 6 (see $[9,13])$. Consider the operator $T_{t}$ defined by (9), where $\mathbf{V} \in \Theta$, which is described by (13). Then

(i) $I_{t}=1+t \operatorname{div} \mathbf{V}+t^{2} \operatorname{det} D \mathbf{V}$,

(ii) there exist $t_{V}, \alpha_{1}, \alpha_{2}>0$ such that $0<\alpha_{1} \leq I_{t}(x) \leq \alpha_{2}$, for $|t| \leq t_{V}, x \in U$.

Proof. In general, for $n$-dimensional case, the Jacobian of $T_{t}$ is given by $D T_{t}=\left(a_{i j}\right)$, where $a_{i j}=t\left(\partial V_{i} / \partial x_{j}\right)$ if, $i \neq j$, and $a_{i i}=1+t\left(\partial V_{i} / \partial x_{i}\right)$. By definition of the determinant, we can write $\operatorname{det} D T_{t}$ as

$$
\begin{aligned}
\operatorname{det} D T_{t} & =\sum_{\sigma \in S_{n}} \operatorname{sgn}(\sigma) a_{1, \sigma(1)} a_{2, \sigma(2)} \cdots a_{n, \sigma(n)} \\
& =\prod_{i=1}^{n}\left(1+t \frac{\partial V_{i}}{\partial x_{i}}\right)
\end{aligned}
$$

$$
\begin{aligned}
&+\sum_{\sigma \in F_{n} \backslash\{i\}} \operatorname{sgn}(\sigma) a_{1, \sigma(1)} a_{2, \sigma(2)} \cdots a_{n, \sigma(n)} \\
&+\sum_{\sigma \in S_{n} \backslash F_{n}} \operatorname{sgn}(\sigma) a_{1, \sigma(1)} a_{2, \sigma(2)} \cdots a_{n, \sigma(n)} \\
&=: I+I I+I I I,
\end{aligned}
$$

where $S_{n}$ refers to the set of all permutations of $\{1,2, \ldots, n\}$, $i$ is the identity permutation, $F_{n}=\left\{\sigma \in S_{n}: \sigma(k)=k\right.$ for some positive integer $k \leq n\}$, and $\operatorname{sgn}(\sigma)$ is either 1 (if the number of inversions is even) or -1 (if the number of inversions is odd). We observe that the expression $I$ can be written as $I=1+t \operatorname{div} V+t^{2} R_{1}(t, \mathbf{V})$, where $R_{1} \in$ $C\left(\mathbb{R}, C^{0,1}(U)\right)$. We also observe that, for $n \geq 2$, each term of the expression $I I$ has at least 2 factors that are of the form $t\left(\partial V_{i} / \partial x_{j}\right), i \neq j$. Hence we can write $I I=t^{2} R_{2}(t, \mathbf{V})$, where $R_{2}$ is in $C\left(\mathbb{R}, C^{0,1}(U)\right)$. All terms of $I I I$ have factors of the form $t\left(\partial V_{i} / \partial x_{j}\right), i \neq j$, and thus we have $I I I=t^{n} \bar{R}_{3}(\mathbf{V})$, which can be written as $t^{2} R_{3}(t, \mathbf{V})$, where $R_{3} \in C\left(\mathbb{R}, C^{0,1}(U)\right)$. Combining $I, I I$, and $I I I$, we get

$$
\operatorname{det} D T_{t}=1+t \operatorname{div} \mathbf{V}+t^{2} R(t, \mathbf{V})
$$

with $R \in C\left(\mathbb{R}, C^{0,1}(U)\right)$. In particular, for $n=2$, the determinant is computed as follows:

$$
\operatorname{det} D T_{t}=1+t \operatorname{div} \mathbf{V}+t^{2} \operatorname{det} D \mathbf{V}
$$

This verifies (i). To show (ii) we first get the lower bound for $I_{t}(x)$. Take

$$
t_{V}=\min \left\{\frac{1}{2\left(|\operatorname{div} \mathbf{V}|_{C(\bar{U})}+|\operatorname{det} D \mathbf{V}|_{C(\bar{U})}\right)}, 1\right\} .
$$

For $|t| \leq t_{V}$, we obtain

$$
\begin{aligned}
I_{t}(x) & \geq 1-|t||\operatorname{div} \mathbf{V}|_{C(\bar{U})}-|t|^{2}|\operatorname{det} D \mathbf{V}|_{C(\bar{U})} \\
& \geq 1-|t||\operatorname{div} \mathbf{V}|_{C(\bar{U})}-|t||\operatorname{det} D \mathbf{V}|_{C(\bar{U})} \\
& =1-|t|\left(|\operatorname{div} \mathbf{V}|_{C(\bar{U})}+|\operatorname{det} D \mathbf{V}|_{C(\bar{U})}\right) \geq \frac{1}{2} .
\end{aligned}
$$

On the other hand, by triangle inequality we have

$$
\begin{array}{r}
I_{t}(x) \leq 1+|t||\operatorname{div} \mathbf{V}|_{C(\bar{U})}+|t|^{2}|\operatorname{det} D \mathbf{V}|_{C(\bar{U})} \\
\leq 1+t_{V}|\operatorname{div} \mathbf{V}|_{C(\bar{U})}+t_{V}^{2}|\operatorname{det} D \mathbf{V}|_{C(\bar{U})} \\
\forall x \in \bar{U} .
\end{array}
$$

Hence, we have shown that there are positive constants $\alpha_{1}=$ $1 / 2$ and $\alpha_{2}=1+t_{V}|\operatorname{div} \mathbf{V}|_{C(\bar{U})}+t_{V}^{2}|\operatorname{det} D V|_{C(\bar{U})}$ such that $\alpha_{1} \leq I_{t}(x) \leq \alpha_{2}$ for $x \in \bar{U}$.

Considering the theorems and lemmas presented beforehand, we are now ready to prove the following theorem. 
Theorem 7. Let $\Omega$ and $U$ be nonempty bounded open connected subsets of $\mathbb{R}^{2}$ with Lipschitz continuous boundaries, such that $\bar{\Omega} \subseteq U$, and $\partial \Omega$ is the union of two disjoint boundaries $\Gamma$ and $\Sigma$. Let $T_{t}$ be defined as in (9) where $\mathbf{V}$ belongs to $\Theta$, defined as (13).

Then for sufficiently small $t$,

(1) $T_{t}: \bar{U} \rightarrow \bar{U}$ is a homeomorphism,

(2) $T_{t}: U \rightarrow U$ is a $C^{1,1}$ diffeomorphism, and in particular, $T_{t}: \Omega \rightarrow \Omega_{t}$ is a $C^{1,1}$ diffeomorphism,

(3) $\Gamma_{t}=T_{t}(\Gamma)=\Gamma$,

(4) $\partial \Omega_{t}=\Gamma \cup T_{t}(\Sigma)$.

Proof. First, because $U$ is a $C^{0,1}$ domain, it follows that int $\bar{U}=$ $U$ by Theorem 2 . Second, $T_{0}=I \in C\left(\bar{U}, \mathbb{R}^{2}\right)$, and it is injective. Third, it is evident that $T_{t}$ is $C^{1,1}$ because $\mathbf{V}$ is $C^{1,1}$. For $x \in \partial U, T_{t}(x)=x$ because $\mathbf{V}$ vanishes on $\partial U$. For $x \in U$, the determinant of the Jacobian of the perturbation of identity operator $T_{t}$ is given by (19). By Lemma 6 , there exists a $t_{V}>0$, given by (20), such that $\operatorname{det} D T_{t}(x)>0$ for all $x \in U$ and for $|t| \leq t_{V}$. Hence, by applying Theorem 4 , we conclude that $T_{t}(U)=U$ and $T_{t}(\bar{U})=\bar{U}$ for all $|t|<t_{V}$, and $T_{t}: \bar{U} \rightarrow \bar{U}$ is a homeomorphism. Furthermore, by Theorem 4 , we find that $T_{t}: U \rightarrow U$ is a $C^{1}$ diffeomorphism. To show that $T_{t}$ is a $C^{1,1}$ diffeomorphism, we are left to show that $D T_{t}^{-1}$ is Lipschitz continuous. To verify this we use Lemma 5.

Given any two points $u, v \in U$ we choose $\left\{y_{k}\right\}$ such that properties (a)-(c) of Lemma 5 are satisfied. For fixed $|t|<t_{V}$, differentiating the identities $T_{t} \circ T_{t}^{-1}=I$ and $T_{t}^{-1} \circ T_{t}=I$ will lead to

$$
\begin{aligned}
& D T_{t}\left(T_{t}^{-1}(z)\right) D T_{t}^{-1}(z)=I, \\
& D T_{t}^{-1}(z) D T_{t}\left(T_{t}^{-1}(z)\right)=I,
\end{aligned}
$$

for all $z \in U$. Thus,

$$
\begin{gathered}
D T_{t}\left(T_{t}^{-1}\left(y_{k+1}\right)\right) D T_{t}^{-1}\left(y_{k+1}\right)=I \\
D T_{t}^{-1}\left(y_{k}\right) D T_{t}\left(T_{t}^{-1}\left(y_{k}\right)\right)=I
\end{gathered}
$$

This implies

$$
\begin{aligned}
& \left|D T_{t}^{-1}(u)-D T_{t}^{-1}(v)\right| \\
& \leq \sum_{k=1}^{l}\left|D T_{t}^{-1}\left(y_{k}\right)-D T_{t}^{-1}\left(y_{k+1}\right)\right| \\
& =\sum_{k=1}^{l} \mid D T_{t}^{-1}\left(y_{k}\right) \\
& \quad \times\left[D T_{t}\left(T_{t}^{-1}\left(y_{k+1}\right)\right)-D T_{t}\left(T_{t}^{-1}\left(y_{k}\right)\right)\right] \\
& \quad \times D T_{t}^{-1}\left(y_{k+1}\right) \mid .
\end{aligned}
$$

Applying the infinity norm in the space $C\left(X ; \mathbb{R}^{2}\right)$ we have

$$
\begin{aligned}
& \left|D T_{t}^{-1}(u)-D T_{t}^{-1}(v)\right| \\
& \leq \sum_{k=1}^{l}\left|D T_{t}^{-1}\left(y_{k}\right)\right|\left|D T_{t}^{-1}\left(y_{k+1}\right)\right| \\
& \quad \times\left|D T_{t}\left(T_{t}^{-1}\left(y_{k+1}\right)\right)-D T_{t}\left(T_{t}^{-1}\left(y_{k}\right)\right)\right| \\
& \leq \max _{y \in \bar{U}}\left|D T_{t}^{-1}(y)\right|^{2} \\
& \quad \times \sum_{k=1}^{l}\left|D T_{t}\left(T_{t}^{-1}\left(y_{k+1}\right)\right)-D T_{t}\left(T_{t}^{-1}\left(y_{k}\right)\right)\right| .
\end{aligned}
$$

Since $D T_{t}$ is Lipschitz continuous, we have

$$
\begin{aligned}
& \left|D T_{t}^{-1}(u)-D T_{t}^{-1}(v)\right| \\
& \quad=\max _{y \in \bar{U}}\left|D T_{t}^{-1}(y)\right|^{2} L_{1} \sum_{k=1}^{l}\left|T_{t}^{-1}\left(y_{k+1}\right)-T_{t}^{-1}\left(y_{k}\right)\right|,
\end{aligned}
$$

where $L_{1}$ is the maximum of all Lipschitz constants of $D T_{t}$ for all $|t|<t_{V}$. Then finally, using the mean value theorem and property (c) in Lemma 5, we obtain

$$
\begin{aligned}
\left|D T_{t}^{-1}(u)-D T_{t}^{-1}(v)\right| & \leq \max _{y \in \bar{U}}\left|D T_{t}^{-1}(y)\right|^{3} L_{1} \sum_{k=1}^{l}\left|y_{k+1}-y_{k}\right| \\
& \leq \max _{y \in \bar{U}}\left|D T_{t}^{-1}(y)\right|^{3} L_{1} c(U)|u-v| .
\end{aligned}
$$

Hence $D T_{t}^{-1}$ is Lipschitz continuous which shows that $T_{t}$ : $U \rightarrow U$ is a $C^{1,1}$ diffeomorphism for sufficiently small $|t|$. Restricting to $\Omega$, this proves that $T_{t}: \Omega \rightarrow \Omega_{t}$ is a $C^{1,1}$ diffeomorphism. (2) is clear because the fixed boundary is invariant under $T_{t}$; that is, $\Gamma_{t}:=T_{t}(\Gamma)=\Gamma$ since $\mathbf{V}$ vanishes on $\Gamma$. Lastly, using Theorem 3 , definition of $T_{t},(1)$, and (2), we obtain (3).

Corollary 8. Let $\Omega$ and $U$ be two domains of $\mathbb{R}^{2}$ with $C^{1,1}$ boundary. Then for $|t|<t_{V}$, where $t_{V}$ is given by (20), the perturbed domain $\Omega_{t}:=T_{t}(\Omega)$ is also of class $C^{1,1}$.

Proof. Given $y \in \partial \Omega_{t}$, we let $x=T_{t}^{-1}(y) \in \partial \Omega$. Then there exists a neighborhood $N_{x}$ of $x$ and a diffeomorphism $g_{x} \in C^{1,1}\left(N_{x}, B(0,1)\right)$ such that $g_{x}\left(N_{x} \cap\right.$ $\Omega)=B^{+}(0,1), g_{x}\left(N_{x} \cap \partial \Omega\right)=B^{0}(0,1)$, and $g_{x}\left(N_{x} \cap\right.$ $\left.\Omega^{c}\right)=B^{-}(0,1)$. We have also shown that $T_{t}$ defined in Theorem 7 is a $C^{1,1}$ diffeomorphism. Since $T_{t}^{-1}$ is continuous, $T_{t}\left(N_{x}\right)=\left(T_{t}^{-1}\right)^{-1}\left(N_{x}\right)=N_{y}$ is a neighborhood of $y$ in $U$. Define $g_{y}:=g_{x} \circ T_{t}^{-1}$. This is bijective because $g_{x}$ and $T_{t}^{-1}$ are bijective. $g_{y} \in C^{1,1}\left(N_{y}, B(0,1)\right)$ because $T_{t}^{-1} \in C^{1,1}(\bar{U}, \bar{U})$ (hence $\left.T_{t}^{-1} \in C^{1,1}\left(N_{y}, N_{x}\right)\right)$ and $g_{x} \in$ $C^{1,1}\left(N_{x}, B(0,1)\right)$. Also, $g_{y}^{-1}=T_{t} \circ g_{x}^{-1} \in C^{1,1}\left(B(0,1), N_{y}\right)$. 


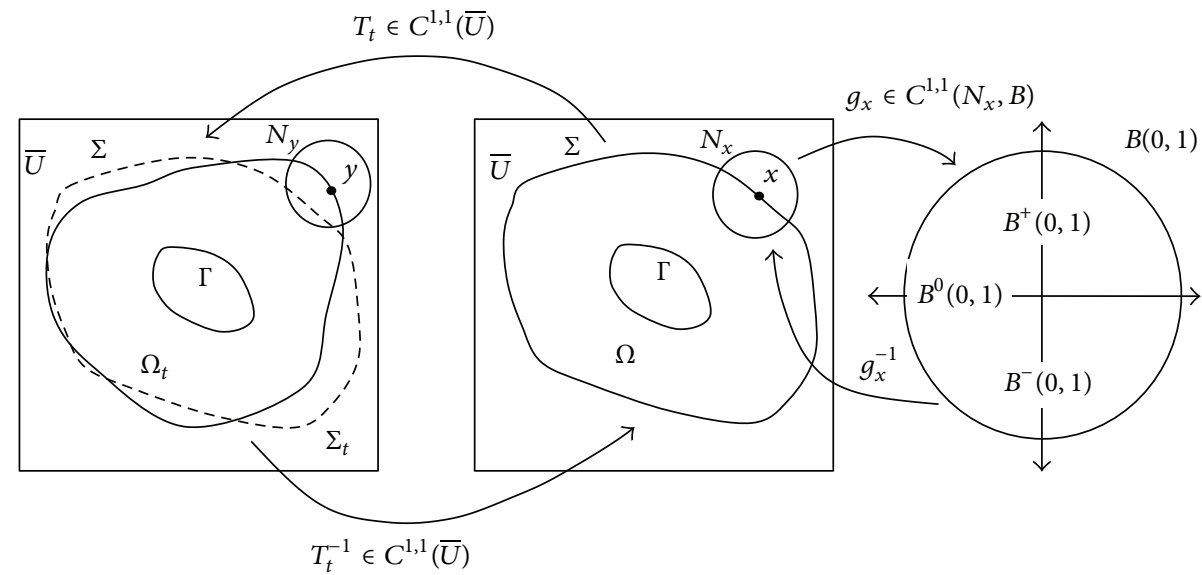

$$
\begin{aligned}
& N_{y} \underset{T_{t}^{-1} \in C^{1,1}}{\longrightarrow} N_{x} \underset{g_{x} \in C^{1,1}}{\longrightarrow} B \\
& N_{y} \underset{T_{t} \in C^{1,1}}{\longleftarrow} N_{x} \underset{g_{x}^{-1} \in C^{1,1}}{\longleftarrow} B
\end{aligned}
$$

FIgURE 3: The action of $T_{t}$ on a $C^{1,1}$-domain.

Next, we note that $\partial \Omega_{t} \cap N_{y}=T_{t}(\partial \Omega) \cap N_{y}=T_{t}(\partial \Omega) \cap$ $T_{t}\left(N_{x}\right)$. Since $T_{t}$ is injective, we have $\partial \Omega_{t} \cap N_{y}=T_{t}\left(\partial \Omega \cap N_{x}\right)$. Thus by definition of $g_{y}$ we get

$$
\begin{aligned}
g_{y}\left(\partial \Omega_{t} \cap N_{y}\right) & =g_{y}\left(T_{t}\left(\partial \Omega \cap N_{x}\right)\right) \\
& =g_{x}\left(\partial \Omega \cap N_{x}\right)=B^{0}(0,1) .
\end{aligned}
$$

We also observe the following:

$$
\begin{aligned}
g_{y}\left(\Omega_{t} \cap N_{y}\right) & =g_{y}\left(T_{t}\left(\Omega \cap N_{x}\right)\right) \\
& =g_{x}\left(\Omega \cap N_{x}\right) \\
& =B^{+}(0,1), \\
g_{y}\left(\bar{\Omega}_{t}^{c} \cap N_{y}\right) & =g_{y}\left(T_{t}\left(\bar{\Omega}^{c} \cap N_{x}\right)\right) \\
& =g_{x}\left(\bar{\Omega}^{c} \cap N_{x}\right) \\
& =B^{-}(0,1) .
\end{aligned}
$$

This shows that $\Omega_{t}$ is indeed of class $C^{1,1}$.

Remark 9. Theorem 7 and Corollary 8 tell us that the reference $\Omega$ and the perturbed domain $\Omega_{t}$ have the same topological structure and regularity under the perturbation of identity operator $T_{t}$ for sufficiently small $t$. See Figure 3 for illustration.

Properties of $T_{t}$. In addition to (16) we also use the following notations throughout the work:

$$
\begin{gathered}
M_{t}(x)=\left(D T_{t}(x)\right)^{-T}, \quad x \in \bar{U}, \\
A_{t}(x)=I_{t}(x) M_{t}^{T}(x) M_{t}(x), \quad x \in \bar{U}, \\
w_{t}(x)=I_{t}(x)\left|\left(D T_{t}(x)\right)^{-T} \mathbf{n}(x)\right|, \quad x \in \Sigma .
\end{gathered}
$$

Remark 10. We note the following observations for fixed, sufficiently small $t$.

(1) $I_{t} \in C^{0,1}(\bar{U})$.

(2) $M_{t}, M_{t}^{T} \in C\left(\bar{U} ; \mathbb{R}^{2 \times 2}\right)$.

(3) $A_{t} \in C\left(\bar{U} ; \mathbb{R}^{2 \times 2}\right)$.

(4) $w_{t} \in C(\Sigma ; \mathbb{R})$.

(5) $\mathbf{V} \in C^{1,1}\left(\bar{U} ; \mathbb{R}^{2}\right)$ implies that $|\mathbf{V}|_{\infty}$ and $|D \mathbf{V}|_{\infty}$ are both finite.

We now provide several properties of $T_{t}$.

Lemma 11 (see $[9,13,16,18]$ ). Consider the transformation $T_{t}$, where the fixed vector field $\mathbf{V}$ belongs to $\Theta$, defined in (13). Then there exists $t_{V}>0$ such that $T_{t}$ and the functions in (16) and (31) restricted to the interval $I_{V}=\left(-t_{V}, t_{V}\right)$ have the following regularity and properties.

(1) $t \mapsto T_{t} \in C^{1}\left(I_{V}, C^{1,1}\left(\bar{U}, \mathbb{R}^{2}\right)\right)$.

(2) $t \mapsto I_{t} \in C^{1}\left(I_{V}, C^{0,1}(\bar{U})\right)$.

(3) $t \mapsto T_{t}^{-1} \in C\left(I_{V}, C^{1}\left(\bar{U}, \mathbb{R}^{2}\right)\right)$.

(4) $t \mapsto w_{t} \in C^{1}\left(I_{V}, C(\Sigma)\right)$.

(5) $t \mapsto A_{t} \in C\left(I_{V}, C\left(\bar{U}, \mathbb{R}^{2 \times 2}\right)\right)$.

(6) There is $\beta>0$ such that $A_{t}(x) \geq \beta I$ for $x \in U$.

(7) $\left.(d / d t) T_{t}\right|_{t=0}=\mathbf{V}$.

(8) $\left.(d / d t) T_{t}^{-1}\right|_{t=0}=-\mathbf{V}$.

(9) $\left.(d / d t) D T_{t}\right|_{t=0}=D V$.

(10) $\left.(d / d t)\left(D T_{t}\right)^{-1}\right|_{t=0}=-D \mathbf{V}$.

(11) $\left.(d / d t) I_{t}\right|_{t=0}=\operatorname{div} \mathbf{V}$.

(12) $\left.(d / d t) A_{t}\right|_{t=0}=(\operatorname{div} \mathbf{V}) I-\left(D \mathbf{V}+(D \mathbf{V})^{T}\right) \equiv A$ 
(13) $\lim _{t \rightarrow 0} w_{t}=1$.

(14) $\left.(d / d t) w_{t}\right|_{t=0}=\operatorname{div}_{\Sigma} \mathbf{V}$,

where the surface divergence $\operatorname{div}_{\Sigma}$ is defined by

$$
\operatorname{div}_{\Sigma} \mathbf{V}=\left.\operatorname{div} \mathbf{V}\right|_{\Sigma}-(D \mathbf{V n}) \cdot \mathbf{n} .
$$

We provide proofs for properties (3) and (8). The rest can be seen in [19].

Proof. (3) Suppose $x \in \bar{U}, t, h \in I_{V}$, and $y=T_{h}^{-1}(x)$. Then

$$
\begin{aligned}
T_{t}^{-1} & (x)-T_{h}^{-1}(x) \\
& =T_{t}^{-1}\left(T_{h}(y)\right)-T_{h}^{-1}\left(T_{h}(y)\right) \\
& =T_{t}^{-1}\left(T_{h}(y)\right)-T_{t}^{-1}\left(T_{t}(y)\right) .
\end{aligned}
$$

Using Lemma 5 , we connect $T_{h}(y)$ and $T_{t}(y)$ by a chain $y_{k}$, $k=1, \ldots, l+1$, satisfying

(i) $y_{1}=T_{t}(y), y_{k} \in U$ for $2 \leq k \leq l, y_{l+1}=T_{h}(y)$,

(ii) $\left(y_{k}, y_{k+1}\right) \subset U$ for $1 \leq k \leq l$,

(iii) $\sum_{k=1}^{l}\left|y_{k+1}-y_{k}\right| \leq c(U)\left|T_{h}(y)-T_{t}(y)\right|$,

and then we get

$$
\begin{aligned}
& \left|T_{t}^{-1}(x)-T_{h}^{-1}(x)\right| \\
& \quad \leq \sum_{k=1}^{l}\left|T_{t}^{-1}\left(y_{k}\right)-T_{t}^{-1}\left(y_{k+1}\right)\right| \\
& \quad \leq \max _{u \in \bar{U}}\left|D T_{t}^{-1}(u)\right| \sum_{k=1}^{l}\left|y_{k}-y_{k+1}\right| \\
& \quad \leq c(U) \max _{u \in \bar{U}}\left|D T_{t}^{-1}(u)\right|\left|T_{h}(y)-T_{t}(y)\right|, \\
& \quad \leq c(U) \max _{u \in \bar{U}}\left|D T_{t}^{-1}(u)\right||\mathbf{V}(y)||t-h| .
\end{aligned}
$$

Thus,

$$
\max _{x \in \bar{U}}\left|T_{t}^{-1}(x)-T_{h}^{-1}(x)\right| \leq c(U) \max _{u \in \bar{U}}\left|D T_{t}^{-1}(u)\right||\mathbf{V}|_{\infty}|t-h| .
$$

By reducing $t_{V}$ if necessary, we can assume without loss of generality that $|t D \mathbf{V}|_{\infty}<1$ for $t \in\left(-t_{V}, t_{V}\right)$. This allows us to represent $\left(D T_{t}\right)^{-1}$ as a Neumann series:

$$
D T_{t}^{-1}(x)=\left(D T_{t}\right)^{-1} \circ T_{t}^{-1}(x)=\sum_{k=0}^{\infty}(-t)^{k}(D \mathbf{V})^{k}\left(T_{t}^{-1}(x)\right),
$$

and its norm is estimated as follows:

$$
\begin{aligned}
\left|D T_{t}^{-1}(x)\right|_{\infty} & \leq \sum_{k=0}^{\infty}\left|(-t)^{k}(D \mathbf{V})^{k}\left(T_{t}^{-1}(x)\right)\right|_{\infty} \\
& =\frac{1}{1-|t||D \mathbf{V}|_{\infty}} \\
& <\frac{1}{1-t_{V}|D \mathbf{V}|_{\infty}}=: M .
\end{aligned}
$$

This shows uniform convergence in $t \in I_{V}$ and $x \in \bar{U}$. Hence, for every $\epsilon>0$ one can choose a $\delta:=\epsilon / c(U) M|\mathbf{V}|_{\infty}>0$ which implies that, for every $t, h \in I_{V},\left|T_{t}^{-1}-T_{h}^{-1}\right|_{\infty}<\epsilon$ whenever $|t-h|<\delta$. In other words, $t \mapsto T_{t}^{-1} \in C\left(I_{V}\right.$, $\left.C\left(\bar{U}, \mathbb{R}^{2}\right)\right)$.

To show that $t \mapsto T_{t}^{-1}$ is continuous from $I_{V}$ to $C^{1}(\bar{U}$, $\mathbb{R}^{2}$ ), we only need to show that for every $t \in I_{V}$, $\left|D T_{t}^{-1}-D T_{h}^{-1}\right|_{\infty}<\epsilon$ whenever $|t-h|<\delta$ and $h \in I_{V}$. Let $z \in \bar{U}$. Using (37), estimate $\left|D T_{t}^{-1}(z)-D T_{h}^{-1}(z)\right|$ as follows:

$$
\begin{gathered}
\left|D T_{t}^{-1}(z)-D T_{h}^{-1}(z)\right| \\
=\mid\left(D T_{t}\right)^{-1}\left(T_{t}^{-1}(z)\right) \\
-\left(D T_{h}\right)^{-1}\left(T_{h}^{-1}(z)\right) \mid \\
=\mid\left(D T_{t}\right)^{-1}\left(T_{t}^{-1}(z)\right) \\
\times\left(\left(D T_{h}\right)\left(T_{h}^{-1}(z)\right)\right. \\
\left.-\left(D T_{t}\right)\left(T_{t}^{-1}(z)\right)\right) \\
\times\left(D T_{h}\right)^{-1}\left(T_{h}^{-1}(z)\right) \mid \\
\leq M^{2} \mid\left(D T_{h}\right)\left(T_{h}^{-1}(z)\right) \\
-\left(D T_{t}\right)\left(T_{t}^{-1}(z)\right) \mid .
\end{gathered}
$$

Using the definition of Jacobian of a transformation and the regularity of $\mathbf{V}$, we further simplify (38) as follows:

$$
\begin{aligned}
&\left|D T_{t}^{-1}(z)-D T_{h}^{-1}(z)\right| \\
& \leq M^{2}\left|h D \mathbf{V}\left(T_{h}^{-1}(z)\right)-t D \mathbf{V}\left(T_{t}^{-1}(z)\right)\right| \\
& \leq M^{2}|h-t|\left|D \mathbf{V}\left(T_{s}^{-1}(z)\right)\right| \\
& \quad+M^{2}|t|\left|D \mathbf{V}\left(T_{h}^{-1}(z)\right)-D \mathbf{V}\left(T_{t}^{-1}(z)\right)\right| \\
& \leq M^{2} K|h-t|+M^{2} t_{V} L\left|T_{h}^{-1}(z)-T_{t}^{-1}(z)\right|,
\end{aligned}
$$

where $L$ is the Lipshitz constant for $D \mathbf{V}$ and $K$ is upper bound for $|D \mathbf{V}|_{\infty}$. Taking the maximum of both sides of the inequality for all $z \in \bar{U}$ and using (34) we get

$$
\left|D T_{t}^{-1}-D T_{h}^{-1}\right|_{\infty} \leq \alpha|h-t|,
$$

where $\alpha=M^{2} K+M^{2} t_{V} L c(U) M|\mathbf{V}|_{\infty}$. Thus, for any $\epsilon>0$, we choose $\delta=\epsilon / \alpha$, so that if $0<|h-t|<\delta$, then $\left|D T_{t}^{-1}-D T_{h}^{-1}\right|_{\infty}<\epsilon$. Therefore, $t \mapsto T_{t}^{-1} \in C\left(I_{V}, C^{1}(\bar{U}\right.$, $\left.\mathbb{R}^{2}\right)$ ).

Proof of property (8) in Lemma 11 is as follows. Given $x \in$ $\bar{U}$, we have $T_{t}\left(T_{t}^{-1}(x)\right)=x$. This implies that

$$
T_{t+h}\left(T_{t+h}^{-1}(x)\right)-T_{t}\left(T_{t}^{-1}(x)\right)=0 .
$$


Manipulating the left hand side of (41), we get

$$
\begin{aligned}
& \left(T_{t+h}\left(T_{t+h}^{-1}(x)\right)-T_{t}\left(T_{t+h}^{-1}(x)\right)\right) \\
& \quad+\left(T_{t}\left(T_{t+h}^{-1}(x)\right)-T_{t}\left(T_{t}^{-1}(x)\right)\right)=: A+B .
\end{aligned}
$$

We first work on $A$. Applying the definition of $T_{t}$, we get

$$
\begin{aligned}
A= & T_{t+h}^{-1}(x)+(t+h) \mathbf{V}\left(T_{t+h}^{-1}(x)\right) \\
& -T_{t+h}^{-1}(x)-t \mathbf{V}\left(T_{t+h}^{-1}(x)\right) \\
= & h \mathbf{V}\left(T_{t+h}^{-1}(x)\right) .
\end{aligned}
$$

Thus,

$$
\lim _{h \rightarrow 0} \frac{1}{h} A=\mathbf{V}\left(T_{t}^{-1}(x)\right)
$$

Similarly, we can write $B$ as follows:

$$
B=T_{t+h}^{-1}(x)-T_{t}^{-1}(x)+t \mathbf{V}\left(T_{t+h}^{-1}(x)\right)-t \mathbf{V}\left(T_{t}^{-1}(x)\right) .
$$

Hence, we have

$$
\begin{aligned}
\frac{1}{h} B= & \frac{1}{h}\left(T_{t+h}^{-1}(x)-T_{t}^{-1}(x)\right) \\
& +t \frac{1}{h}\left(\mathbf{V}\left(T_{t+h}^{-1}(x)\right)-\mathbf{V}\left(T_{t}^{-1}(x)\right)\right)
\end{aligned}
$$

Suppose $v$ is a coordinate function of $\mathbf{V}$. By the mean value theorem, we observe that

$$
\begin{aligned}
& \frac{1}{h}\left(v\left(T_{t+h}^{-1}(x)\right)-v\left(T_{t}^{-1}(x)\right)\right) \\
& \quad=D v(c) \frac{1}{h}\left(T_{t+h}^{-1}(x)-T_{t}^{-1}(x)\right),
\end{aligned}
$$

where $c$ is a point on the segment joining $T_{t}^{-1}(x)$ and $T_{t+h}^{-1}(x)$, and as $h$ tends to infinity, (47) tends to $(D v)(d / d t) T_{t}^{-1}(x)$. Thus,

$$
\lim _{h \rightarrow 0} \frac{1}{h} B=\frac{d}{d t} T_{t}^{-1}(x)+t D \mathbf{V}\left(T_{t}^{-1}(x)\right) \frac{d}{d t} T_{t}^{-1}(x)
$$

Combining (44) and (48), we get

$$
\mathbf{V}\left(T_{t}^{-1}(x)\right)+\left(I+t D \mathbf{V}\left(T_{t}^{-1}(x)\right)\right) \frac{d}{d t} T_{t}^{-1}(x)=0
$$

which implies that

$$
\frac{d}{d t} T_{t}^{-1}(x)=-\left(I+t D \mathbf{V}\left(T_{t}^{-1}(x)\right)\right)^{-1} \mathbf{V}\left(T_{t}^{-1}(x)\right)
$$

Evaluating (50) at $t=0$, we get $\left.(d / d t) T_{t}^{-1}\right|_{t=0}=-\mathbf{V}$.
3.3. The Method of Mapping. If $u$ is defined in $\Omega$ and $u_{t}$ is defined in $\Omega_{t}$, then the direct comparison of $u_{t}$ with $u$ is generally not possible since the functions are defined on different domains. To overcome this difficulty, one maps $u_{t}$ back to $\Omega$ by composing it with $T_{t}$; that is, one defines $u_{t}$ 。 $T_{t}: \Omega \rightarrow \mathbb{R}$. With this new mapping one can define the material and the shape derivatives of states, the domain and boundary integral transformations, and derivatives of integrals, as well as the Eulerian derivative of the shape functional. This technique is called the method of mapping.

Material and Shape Derivatives. The material and shape derivatives of state variables are defined as follows [20,21].

Definition 12. Let $u$ be defined in $\left[0, t_{V}\right] \times U$. An element $\dot{u} \epsilon$ $H^{k}(\Omega)$, called the material derivative of $u$, is defined as

$$
\begin{aligned}
\dot{u}(x):=\dot{u}(0, x) & :=\lim _{t \rightarrow 0^{+}} \frac{u\left(t, T_{t}(x)\right)-u(0, x)}{t} \\
& =\left.\frac{d}{d t} u(t, x+t \mathbf{V}(x))\right|_{t=0}
\end{aligned}
$$

if the limit exists in $\left(H^{k}(\Omega)\right)$.

Remark 13. The material derivative can be written as

$$
\dot{u}(x)=\lim _{t \rightarrow 0^{+}} \frac{u_{t} \circ T_{t}(x)-u(x)}{t}=\left.\frac{d}{d t}\left(u_{t} \circ T_{t}(x)\right)\right|_{t=0} .
$$

It characterizes the behavior of the function $u$ at $x \in \Omega \subset U$ in the direction $\mathbf{V}(x)$.

Definition 14. Let $u$ be defined in $\left[0, t_{V}\right] \times U$. An element $u^{\prime} \in$ $H^{k}(\Omega)$ is called the shape derivative of $u$ at $\Omega$ in the direction $\mathbf{V}$, if the following limit exists in $H^{k}(\Omega)$ :

$$
\begin{aligned}
u^{\prime}(x) & :=u^{\prime}(0, x) \\
& :=\lim _{t \rightarrow 0^{+}} \frac{u(t, x)-u(0, x)}{t} .
\end{aligned}
$$

Remark 15. The shape derivative of $u$ is also defined as follows:

$$
u^{\prime}(x):=\left.\frac{\partial}{\partial t} u_{t}(x)\right|_{t=0}
$$

We note that if $\dot{u}$ and $\nabla u \cdot V$ exist in $H^{k}(\Omega)$, then the shape derivative can be written as

$$
u^{\prime}(x)=\dot{u}(x)-(\nabla u \cdot \mathbf{V})(x) .
$$

In general, if $\dot{u}(x)$ and $\nabla u \cdot V(x)$ both exist in $W^{m, p}(\Omega)$, then $u^{\prime}(x)$ also exists in that space.

\section{Domain and Boundary Transformations}

Lemma 16 (see [18]). (1) Let $\varphi_{t} \in L^{1}\left(\Omega_{t}\right)$. Then $\varphi_{t} \circ T_{t} \in L^{1}(\Omega)$ and

$$
\int_{\Omega_{t}} \varphi_{t} d x_{t}=\int_{\Omega} \varphi_{t} \circ T_{t} I_{t} d x
$$


(2) Let $\varphi_{t} \in L^{1}\left(\partial \Omega_{t}\right)$. Then $\varphi_{t} \circ T_{t} \in L^{1}(\partial \Omega)$ and

$$
\int_{\partial \Omega_{t}} \varphi_{t} d s_{t}=\int_{\partial \Omega} \varphi_{t} \circ T_{t} w_{t} d s
$$

where $I_{t}$ and $w_{t}$ are defined in (31).

Proofs can be found in $[13,18]$.

Domain and Boundary Differentiation. We recall some results concerning the derivative of integrals with respect to the domain of integration. For the first theorem, it is sufficient to have $C^{0,1}$ domains while the second theorem requires $C^{1,1}$ domains. For proofs, see [18].

Theorem 17 (domain differentiation formula). Let $u \in C\left(I_{V}\right.$, $\left.W^{1,1}(U)\right)$ and suppose $\dot{u}(0, \cdot):=\left.(d / d t) u\left(t, T_{t}(\cdot)\right)\right|_{t=0}$ exists in $L^{1}(U)$. Then

$$
\left.\frac{d}{d t} \int_{\Omega_{t}} u(t, x) d x\right|_{t=0}=\int_{\Omega} u^{\prime}(0, x) d x+\int_{\Sigma} u(0, s) \mathbf{V} \cdot \mathbf{n} d s
$$

Theorem 18 (boundary differentiation formula). Let $u$ be defined in a neighborhood of $\Gamma$. If $u \in C\left(I_{V}, W^{2,1}(U)\right)$ and $\dot{u}(0, \cdot) \in W^{1,1}(U)$, then

$$
\begin{aligned}
& \left.\frac{d}{d t} \int_{\Sigma_{t}} u(t, s) d s\right|_{t=0} \\
& \quad=\int_{\Sigma} u^{\prime}(0, s) d s+\int_{\Sigma}\left(\frac{\partial u}{\partial \mathbf{n}}+u(0, s) \kappa\right) \mathbf{V} \cdot \mathbf{n} d s
\end{aligned}
$$

where $\kappa$ is the mean curvature of the free boundary $\Sigma$.

\section{The First-Order Eulerian Derivative}

Definition 19. The Eulerian derivative of the shape functional $J: \Omega \rightarrow \mathbb{R}$ defined in (7) at the domain $\Omega$ in the direction of the deformation field $\mathbf{V} \in \Theta$ is given by

$$
d J(\Omega ; \mathbf{V}):=\lim _{t \rightarrow 0^{+}} \frac{J\left(\Omega_{t}\right)-J(\Omega)}{t},
$$

if the limit exists.

Remark 20. $J$ is said to be shape differentiable at $\Omega$ if $d J(\Omega ; \mathbf{V})$ exists for all $\mathbf{V} \in \Theta$ and is linear and continuous with respect to $\mathrm{V}$.

\section{Main Result}

In this section we derive in a rigorous manner the first-order shape derivative of the Kohn-Vogelius functional $J$, defined by (7), subject to the Dirichlet and Neumann boundary value problems (BVPs) (4) and (6), respectively. Our strategy bypasses the material or shape derivatives of states. In the derivation, we have employed techniques used in $[9,10,13]$ but there is no need to use adjoint variables.

This section discusses the variational forms of the PDEs, the state variables in the perturbed domains, the Hölder continuity of the state variables, and the higher regularity of the solutions to the BVPs. The rest of the proof is presented in the last part of this section.

4.1. Variational Forms of the Dirichlet and Neumann Problems. We recall that we are considering the shape optimization problem (7) where $u_{D}$ solves the pure Dirichlet problem (4) and $u_{N}$ solves the Neumann problem (6). As in [13], we consider the Hilbert space

$$
H_{\Gamma, 0}^{1}=\left\{\varphi \in H^{1}(\Omega):\left.\varphi\right|_{\Gamma}=0\right\},
$$

which is endowed with the norm

$$
|\varphi|_{1}^{2}=\int_{\Omega}|\nabla \varphi|^{2} \mathrm{~d} x
$$

and a linear manifold defined by

$$
H_{\Gamma, v}^{1}=\left\{\varphi \in H^{1}(\Omega):\left.\varphi\right|_{\Gamma}=v\right\}
$$

for $v \in H^{1 / 2}(\Gamma)$.

First, we determine the variational equations for the Dirichlet and the Neumann problems. The variational form of the Dirichlet problem (4) is given by the following.

Find $u_{D} \in H^{1}(\Omega)$ such that

$$
\begin{array}{rlrl}
\left(\nabla u_{D}, \nabla \psi\right)_{\Omega} & =0 & & \forall \psi \in H_{0}^{1}(\Omega) \\
u_{D}=1 & & \text { on } \Gamma, \\
u_{D}=0 & & \text { on } \Sigma .
\end{array}
$$

Equation (64) can be shown to have a unique solution using Theorem 2.4.2.5 of [22]. Similarly, the variational form of the Neumann problem (6) is formulated as follows.

Find $u_{N} \in H^{1}(\Omega)$ such that

$$
\begin{gathered}
\left(\nabla u_{N}, \nabla \varphi\right)_{\Omega}-(\lambda, \varphi)_{\Sigma}=0 \quad \forall \varphi \in H_{\Gamma, 0}^{1}(\Omega) \\
u_{N}=1 \quad \text { on } \Gamma .
\end{gathered}
$$

It is also well known that (65) has a unique solution.

4.2. Analysis of State Variables in Deformed Domains. We now consider the class of perturbed problems:

$$
\min _{\Omega_{t}} J\left(\Omega_{t}\right)=\frac{1}{2} \int_{\Omega_{t}}\left|\nabla\left(u_{D, t}-u_{N, t}\right)\right|^{2} d x,
$$

where $u_{D, t}$ solves the pure Dirichlet problem

$$
\begin{array}{cc}
-\Delta u_{D, t}=0 & \text { in } \Omega_{t}, \\
u_{D, t}=1 & \text { on } \Gamma, \\
u_{D, t}=0 & \text { on } \Sigma_{t},
\end{array}
$$

and $u_{N, t}$ solves the Neumann problem

$$
\begin{array}{cc}
-\Delta u_{N, t}=0 & \text { in } \Omega_{t}, \\
u_{N, t}=1 & \text { on } \Gamma, \\
\frac{\partial u_{N, t}}{\partial \mathbf{n}_{t}}=\lambda & \text { on } \Sigma_{t} .
\end{array}
$$


Here, $\mathbf{n}_{t}$ is the outward unit normal to the deformed free boundary $\Sigma_{t}$. The variational form of (67) is formulated as follows.

Find $u_{D, t} \in H^{1}\left(\Omega_{t}\right)$ such that

$$
\begin{aligned}
\left(\nabla u_{D, t}, \nabla \psi_{t}\right)_{\Omega_{t}} & =0 & & \forall \psi_{t} \in H_{0}^{1}\left(\Omega_{t}\right) \\
u_{D, t} & =1 & & \text { on } \Gamma, \\
u_{D, t} & =0 & & \text { on } \Sigma_{t} .
\end{aligned}
$$

It is known that (69) has a unique solution.

Remark 21. The function $u_{t}: \Omega_{t} \rightarrow \mathbb{R}$ can be referred to as the reference domain by composing $u_{t}$ with $T_{t}$; that is,

$$
u^{t}=u_{t} \circ T_{t}: \Omega \longrightarrow \mathbb{R}
$$

and by chain rule of differentiation, we get

$$
\left(\nabla u_{t}\right) \circ T_{t}=\left(D T_{t}\right)^{-T} \nabla u^{t}=: M_{t} \nabla u^{t}
$$

Let $u_{D, t}$ be the solution of (69). Applying Lemma 16 for all $\psi_{t} \in H_{0}^{1}\left(\Omega_{t}\right)$ we have

$$
\begin{aligned}
\left(\nabla u_{D, t}, \nabla \psi_{t}\right)_{\Omega_{t}} & =\int_{\Omega_{t}} \nabla u_{D, t} \cdot \nabla \psi_{t} \\
& =\int_{\Omega} I_{t}\left(\nabla u_{D, t} \circ T_{t}\right) \cdot\left(\nabla \psi_{t} \circ T_{t}\right) .
\end{aligned}
$$

Applying (71) and noting that $\psi^{t} \in H_{0}^{1}(\Omega)$ because $\psi_{t} \in$ $H_{0}^{1}\left(\Omega_{t}\right)$, we obtain

$$
\begin{aligned}
\left(\nabla u_{D, t}, \nabla \psi_{t}\right)_{\Omega_{t}} & =\int_{\Omega} I_{t} M_{t} \nabla u_{D}^{t} \cdot M_{t} \nabla \psi^{t} \\
& =\int_{\Omega} I_{t} M_{t}^{T} M_{t} \nabla u_{D}^{t} \cdot \nabla \psi^{t} \\
& =\left(A_{t} \nabla u_{D}^{t}, \nabla v\right)_{\Omega},
\end{aligned}
$$

where $v=\psi^{t} \in H_{0}^{1}(\Omega)$. Hence, if $u_{D, t}$ solves the variational equation (69), then $u_{D}^{t}=u_{D, t} \circ T_{t}$ satisfies the variational equation,

$$
\left(A_{t} \nabla u_{D}^{t}, \nabla v\right)_{\Omega}=0
$$

for all $v \in H_{0}^{1}(\Omega), u_{D}^{t}=1$ on $\Gamma$, and $u_{D}^{t}=0$ on $\Sigma$.

Now we show that $u_{D}^{t}$ is the unique solution of (74) in $H^{1}(\Omega)$. First, we show that $y^{t}=u_{D}^{t}-u_{D} \in H_{0}^{1}(\Omega)$ is the unique solution to

$$
\left(A_{t} \nabla y^{t}, \nabla v\right)_{\Omega}=-\left(A_{t} \nabla u_{D}, \nabla v\right)_{\Omega}
$$

for all $v \in H_{0}^{1}(\Omega)$. The bilinear form $b_{t}(\cdot, \cdot): H_{0}^{1}(\Omega) \times$ $H_{0}^{1}(\Omega) \rightarrow \mathbb{R}$ defined by

$$
b_{t}\left(y^{t}, v\right)=\int_{\Omega} A_{t} \nabla y^{t} \cdot \nabla v, \quad \forall y^{t}, v \in H_{0}^{1}(\Omega)
$$

is continuous, because

$$
\begin{aligned}
\left|b_{t}\left(y^{t}, v\right)\right| & =\left|\int_{\Omega} A_{t} \nabla y^{t} \cdot \nabla v\right| \\
& \leq\left|A_{t} \nabla y^{t}\right|_{L^{2}(\Omega)}|\nabla v|_{L^{2}(\Omega)} \\
& \leq 2\left|A_{t}\right|_{\infty}\left|\nabla y^{t}\right|_{L^{2}(\Omega)}|\nabla v|_{L^{2}(\Omega)} \\
& =\beta\left|y^{t}\right|_{H^{1}(\Omega)}|v|_{H_{0}^{1}(\Omega)} .
\end{aligned}
$$

The bilinear form is also coercive. To show this we recall that $\lim _{t \rightarrow 0} A_{t}=I$ uniformly on $\bar{\Omega}$. This is equivalent to the statement

$$
\forall \epsilon>0 \exists \delta>0 \quad \text { such that }|t|<\delta \Longrightarrow\left|A_{t}-I\right|_{\infty}<\epsilon .
$$

Let $\epsilon=1 / 2$. So for sufficiently small $t,\left|A_{t}-I\right|_{\infty}<1 / 2$, and

$$
\begin{aligned}
b_{t}\left(y^{t}, y^{t}\right) & =\int_{\Omega} A_{t} \nabla y^{t} \cdot \nabla y^{t} \\
& =\left.\left|\int_{\Omega}\left(A_{t}-I\right) \nabla y^{t} \cdot \nabla y^{t}+\right| \nabla y^{t}\right|^{2} \mid \\
& \geq\left|\nabla y^{t}\right|_{L^{2}(\Omega)}^{2}-\left|A_{t}-I\right|_{\infty}\left|\nabla y^{t}\right|_{L^{2}(\Omega)}^{2} \\
& \geq \frac{1}{2}\left|y^{t}\right|_{H_{0}^{1}(\Omega)}^{2} .
\end{aligned}
$$

So $b_{t}\left(y^{t}, v\right)$ is coercive.

Next, we show that the functional $f: H_{0}^{1}(\Omega) \rightarrow \mathbb{R}$ is bounded:

$$
\begin{aligned}
\left|\langle f, v\rangle_{H^{-1} \times H_{0}^{1}}\right| & =\left|-\int_{\Omega} A_{t} \nabla u_{D} \cdot \nabla v\right| \\
& \leq 2\left|A_{t}\right|_{\infty}\left|\nabla u_{D}\right|_{L^{2}(\Omega)}|\nabla v|_{L^{2}(\Omega)} \\
& =2\left|A_{t}\right|_{\infty}\left|u_{D}\right|_{H_{0}^{1}(\Omega)}|v|_{H_{0}^{1}(\Omega)} \leq C|v|_{H_{0}^{1}(\Omega)} .
\end{aligned}
$$

Therefore, by the Lax-Milgram lemma, $y^{t}=u_{D}^{t}-u_{D}$ is the unique solution to the variational equation

$$
\left(A_{t} \nabla y^{t}, \nabla v\right)_{\Omega}=-\left(A_{t} \nabla u_{D}, \nabla v\right)_{\Omega}, \quad \forall v \in H_{0}^{1}(\Omega) .
$$

This implies the existence of a unique solution $u_{D}^{t}$ of (74) as verified below.

Let $u_{D}^{t}=y^{t}+u_{D}$. Using (81) we obtain

$$
\begin{aligned}
\left(A_{t} \nabla u_{D}^{t}, \nabla v\right)_{\Omega}= & \left(A_{t} \nabla y^{t}, \nabla v\right)_{\Omega}+\left(A_{t} \nabla u_{D}, \nabla v\right)_{\Omega} \\
& \forall v \in H_{0}^{1}(\Omega) \\
= & -\left(A_{t} \nabla u_{D}, \nabla v\right)_{\Omega}+\left(A_{t} \nabla u_{D}, \nabla v\right)_{\Omega}=0 .
\end{aligned}
$$

Thus (74) is satisfied. The boundary conditions are also satisfied because on $\Gamma, y^{t}=0$ and $u_{D}=1$ and on $\Sigma$, both $y^{t}$ and $u_{D}$ are zero. To show uniqueness, we let $u_{D}^{t}$ and $\tilde{u}_{D}^{t}$ be solutions of (74). This implies that there exist $y^{t}$ and $\tilde{y}^{t}$ 
such that $u_{D}^{t}=y^{t}+u_{D}$ and $\widetilde{u}_{D}^{t}=\tilde{y}^{t}+u_{D}$, where $y^{t}$ and $\widetilde{y}^{t}$ are solutions to (81). Taking the difference of $u_{D}^{t}$ and $\widetilde{u}_{D}^{t}$ and considering that solution to (81) is unique, we get $u_{D}^{t}=\widetilde{u}_{D}^{t}$.

Next, we consider (68) whose variational form is formulated as follows.

Find $u_{N, t} \in H^{1}\left(\Omega_{t}\right)$ such that

$$
\begin{gathered}
\left(\nabla u_{N, t}, \nabla \varphi_{t}\right)_{\Omega_{t}}-\left(\lambda, \varphi_{t}\right)_{\Sigma_{t}}=0 \quad \forall \varphi_{t} \in H_{\Gamma, 0}^{1}\left(\Omega_{t}\right), \\
u_{N, t}=1 \quad \text { on } \Gamma .
\end{gathered}
$$

Similarly, if $u_{N, t}$ solves the variational problem (83), then $u_{N}^{t}$ solves the variational equation

$$
\left(A_{t} \nabla u_{N}^{t}, \nabla \phi\right)_{\Omega}-\left(w_{t} \lambda, \phi\right)_{\Sigma}=0, \quad \forall \phi \in H_{\Gamma, 0}^{1}(\Omega),
$$

where $u_{N}^{t}=1$ on $\Gamma$.

As shown before, the bilinear form $b_{t}(\cdot, \cdot): H_{\Gamma, 0}^{1}(\Omega) \times$ $H_{\Gamma, 0}^{1}(\Omega) \rightarrow \mathbb{R}$ defined by

$$
b_{t}\left(z^{t}, v\right)=\int_{\Omega} A_{t} \nabla z^{t} \cdot \nabla v, \quad \forall z^{t}, v \in H_{\Gamma, 0}^{1}(\Omega),
$$

is coercive and continuous. The linear functional $f$ : $H_{\Gamma, 0}^{1}(\Omega) \rightarrow \mathbb{R}$ defined by $\langle f, \phi\rangle=\int_{\Sigma} \lambda w_{t} \phi$ is continuous on $H_{\Gamma, 0}^{1}(\Omega)$ because

$$
\begin{aligned}
\left|\langle f, \phi\rangle_{H^{-1} \times H_{0}^{1}}\right| & =\left|\int_{\Sigma} \lambda w_{t} \phi\right| \leq|\lambda|\left|w_{t}\right|_{\infty} \int_{\Sigma}|\phi| \\
& =|\lambda|\left|w_{t}\right|_{\infty}|\Sigma|^{1 / 2}|\gamma \phi|_{L^{2}(\Sigma)} \\
& \leq|\lambda|\left|w_{t}\right|_{\infty}|\Sigma|^{1 / 2}\|\gamma\||\phi|_{H^{1}(\Omega)} .
\end{aligned}
$$

By the Lax-Milgram lemma, $z^{t}=u_{N}^{t}-1 \in H_{\Gamma, 0}^{1}(\Omega)$ is the unique solution in $H_{\Gamma, 0}^{1}(\Omega)$ of

$$
\int_{\Omega} A_{t} \nabla z^{t} \cdot \nabla \phi=\lambda \int_{\Sigma} w_{t} \phi, \quad \forall \phi \in H_{\Gamma, 0}^{1}(\Omega) .
$$

Let $u_{N}^{t}=z^{t}+1$. Then, by (87), we get

$$
\begin{array}{r}
\left(A_{t} \nabla u_{N}^{t}, \nabla \phi\right)_{\Omega}-\left(w_{t} \lambda, \phi\right)_{\Sigma}=\left(A_{t} \nabla z^{t}, \nabla \phi\right)_{\Omega}-\left(w_{t} \lambda, \phi\right)_{\Sigma}=0 \\
\phi \in H_{\Gamma, 0}^{1}(\Omega) .
\end{array}
$$

Since $z^{t} \in H_{\Gamma, 0}^{1}(\Omega), u_{N}^{t}=z^{t}+1=1$ on $\Gamma$. Uniqueness of $u_{N}^{t}$ follows from the uniqueness of $z^{t}$. Therefore, $u_{N}^{t}$ is the unique solution of the variational problem (84) in $H^{1}(\Omega)$.

4.3. Hölder Continuity of the States. We show that $u_{D}^{t}$ and $u_{N}^{t}$ are Hölder continuous on $t$.

Theorem 22 (see [13]). The solutions $u_{D}^{t}$ of (74) are uniformly bounded in $H^{1}(\Omega)$ for $t \in\left(-t_{V}, t_{V}\right)$ and

$$
\lim _{t \rightarrow 0} \frac{1}{t}\left|u_{D}^{t}-u_{D}\right|_{H^{1}(\Omega)}^{2}=0,
$$

where $u_{D}$ is the weak solution of (4).
Proof. We first prove the uniform boundedness of $u_{D}^{t}$ in $H^{1}(\Omega)$ for $t \in\left(-t_{V}, t_{V}\right)$. Since $u_{D}^{t}-u_{D} \in H_{0}^{1}(\Omega)$, by using coercivity of $A_{t}$ we get

$$
\left(A_{t} \nabla\left(u_{D}^{t}-u_{D}\right), \nabla\left(u_{D}^{t}-u_{D}\right)\right)_{\Omega} \geq \frac{1}{2}\left|\nabla\left(u_{D}^{t}-u_{D}\right)\right|_{H_{0}^{1}(\Omega)}^{2} .
$$

Also, by applying (81), we have

$$
\begin{aligned}
& \left(A_{t} \nabla\left(u_{D}^{t}-u_{D}\right), \nabla\left(u_{D}^{t}-u_{D}\right)\right)_{\Omega} \\
& \quad=-\left(A_{t} \nabla u_{D}, \nabla\left(u_{D}^{t}-u_{D}\right)\right)_{\Omega} \\
& \quad \leq\left|A_{t}\right|_{\infty}\left|u_{D}\right|_{H^{1}(\Omega)}\left|u_{D}^{t}-u_{D}\right|_{H_{0}^{1}(\Omega)} .
\end{aligned}
$$

Therefore,

$$
\left|u_{D}^{t}\right|_{H^{1}(\Omega)} \leq\left|u_{D}^{t}-u_{D}\right|_{H_{0}^{1}(\Omega)}+\left|u_{D}\right|_{H^{1}(\Omega)} \leq \sqrt{t_{V}}+\left|u_{D}\right|_{H^{1}(\Omega)} .
$$

Now we take the difference between the weak form of (4) and the variational equation (74), to get

$$
\begin{aligned}
& \left(A_{t} \nabla u_{D}^{t}, \nabla v\right)_{\Omega}-\left(\nabla u_{D}, \nabla v\right)_{\Omega}=0, \quad \forall v \in H_{0}^{1}(\Omega) \\
& \left(A_{t} \nabla \frac{1}{t}\left(u_{D}^{t}-u_{D}\right), \nabla v\right)_{\Omega}=-\left(\frac{\left(A_{t}-I\right)}{t} \nabla u_{D}, \nabla v\right)_{\Omega} .
\end{aligned}
$$

Note that $u_{D, t} \in H_{\Gamma, 1}^{1}\left(\Omega_{t}\right)$. This implies that $u_{D}^{t}=u_{D, t} \circ T_{t}$ is in $H_{\Gamma, 1}^{1}(\Omega)$. Note also that $u_{D} \in H_{\Gamma, 1}^{1}(\Omega)$. Let $y^{t}=(1 / t)\left(u_{D}^{t}-u_{D}\right)$. So for sufficiently small $t, y^{t} \in H_{0}^{1}(\Omega)$. Now choosing $v=$ $y^{t}$ as a test function and by the uniform coercivity of $A_{t}$ one obtains

$$
\begin{aligned}
\alpha\left|\nabla y^{t}\right|_{L^{2}(\Omega)}^{2} & \leq\left(A_{t} \nabla y^{t}, \nabla y^{t}\right)_{\Omega} \\
& =-\left(\frac{1}{t}\left(A_{t}-I\right) \nabla u_{D}, \nabla y^{t}\right)_{\Omega} \\
& \leq\left|\frac{1}{t}\left(A_{t}-I\right)\right|_{\infty}\left|\nabla u_{D}\right|_{L^{2}(\Omega)}\left|\nabla y^{t}\right|_{L^{2}(\Omega)} .
\end{aligned}
$$

If $\nabla y^{t}=0$, then the inequality above holds. For $\nabla y^{t} \neq 0$ we have

$$
\begin{aligned}
\alpha\left|\nabla y^{t}\right|_{L^{2}(\Omega)}^{2} & \leq\left|\frac{1}{t}\left(A_{t}-I\right) \nabla u_{D}\right|_{L^{2}(\Omega)}\left|\nabla y^{t}\right|_{L^{2}(\Omega)} \\
& \leq\left|\frac{1}{t}\left(A_{t}-I\right)\right|_{\infty}\left|\nabla u_{D}\right|_{L^{2}(\Omega)}\left|\nabla y^{t}\right|_{L^{2}(\Omega)} .
\end{aligned}
$$

Hence,

$$
\left|\frac{1}{t} \nabla\left(u_{D}^{t}-u_{D}\right)\right|_{L^{2}(\Omega)} \leq \frac{1}{\alpha}\left|\frac{1}{t}\left(A_{t}-I\right)\right|_{\infty}\left|\nabla u_{D}\right|_{L^{2}(\Omega)} .
$$

Squaring and multiplying $t$ on both sides of the inequality give us

$$
\frac{1}{t}\left|\nabla\left(u_{D}^{t}-u_{D}\right)\right|_{L^{2}(\Omega)}^{2} \leq t \frac{1}{\alpha^{2}}\left|\frac{1}{t}\left(A_{t}-I\right)\right|_{\infty}^{2}\left|\nabla u_{D}\right|_{L^{2}(\Omega)}^{2} .
$$


Since $\left|(1 / t)\left(A_{t}-I\right)\right|_{\infty}^{2}$ is uniformly bounded in $t$ by Lemma 11,

$$
\lim _{t \rightarrow 0} \frac{1}{t}\left|u_{D}^{t}-u_{D}\right|_{H^{1}(\Omega)}^{2}=\lim _{t \rightarrow 0} \frac{1}{t}\left|\nabla\left(u_{D}^{t}-u_{D}\right)\right|_{L^{2}(\Omega)}^{2}=0
$$

follows.

Theorem 23. The solutions $u_{N}^{t}$ of (84) are uniformly bounded in $H^{1}(\Omega)$ for $t \in\left(-t_{V}, t_{V}\right)$ and

$$
\lim _{t \rightarrow 0} \frac{1}{t}\left|u_{N}^{t}-u_{N}\right|_{H^{1}(\Omega)}^{2}=0
$$

where $u_{N}$ is the solution of (65).

Proof. Subtracting (65) from (84) for all $\varphi \in H_{\Gamma, 0}^{1}(\Omega)$ we get

$$
\begin{aligned}
0 & =\left(A_{t} \nabla u_{N}^{t}, \nabla \varphi\right)_{\Omega}-\left(w_{t} \lambda, \varphi\right)_{\Sigma}-\left(\nabla u_{N}, \nabla \varphi\right)_{\Omega}+(\lambda, \varphi)_{\Sigma} \\
& =\left(A_{t} \nabla u_{N}^{t}-\nabla u_{N}^{t}+\nabla u_{N}^{t}-\nabla u_{N}, \nabla \varphi\right)_{\Omega}-\left(w_{t} \lambda-\lambda, \varphi\right)_{\Sigma}
\end{aligned}
$$

Hence

$$
\begin{aligned}
\left(\nabla\left(u_{N}^{t}-u_{N}\right), \nabla \varphi\right)_{\Omega}= & -\left(\left(A_{t}-I\right) \nabla u_{N}^{t}, \nabla \varphi\right)_{\Omega} \\
& +\left(w_{t} \lambda-\lambda, \varphi\right)_{\Sigma} .
\end{aligned}
$$

Note that $u_{N}^{t}-u_{N}$ belongs to $H_{\Gamma, 0}^{1}(\Omega)$. Hence

$$
\begin{aligned}
\left|u_{N}^{t}-u_{N}\right|_{H^{1}(\Omega)}^{2}= & -\left(\left(A_{t}-I\right) \nabla u_{N}^{t}, \nabla\left(u_{N}^{t}-u_{N}\right)\right)_{\Omega} \\
& +\lambda\left(w_{t}-1, u_{N}^{t}-u_{N}\right)_{\Sigma} .
\end{aligned}
$$

By Cauchy-Schwarz inequality, we obtain

$$
\begin{aligned}
\left|u_{N}^{t}-u_{N}\right|_{H^{1}(\Omega)}^{2} \leq & \left|\left(A_{t}-I\right) \nabla u_{N}^{t}\right|_{L^{2}(\Omega)}\left|\nabla\left(u_{N}^{t}-u_{N}\right)\right|_{L^{2}(\Omega)} \\
& +|\lambda|\left|w_{t}-1\right|_{\infty}\left|u_{N}^{t}-u_{N}\right|_{L^{2}(\Sigma)} .
\end{aligned}
$$

Furthermore, by trace theorem we have $\left|u_{N}^{t}-u_{N}\right|_{L^{2}(\Sigma)} \leq$ $c\left|u_{N}^{t}-u_{N}\right|_{H^{1}(\Omega)}$. Therefore,

$$
\begin{aligned}
& \left|u_{N}^{t}-u_{N}\right|_{H^{1}(\Omega)}^{2} \\
& \quad \leq C\left(\left|\left(A_{t}-I\right) \nabla u_{N}^{t}\right|_{L^{2}(\Omega)}+|\lambda|\left|w_{t}-1\right|_{\infty}\right)\left|u_{N}^{t}-u_{N}\right|_{H^{1}(\Omega)}
\end{aligned}
$$

holds, where $C=\max \{1, c\}$. This implies

$$
\left|u_{N}^{t}-u_{N}\right|_{H^{1}(\Omega)} \leq C\left(\left|\left(A_{t}-I\right) \nabla u_{N}^{t}\right|_{L^{2}(\Omega)}+\left|w_{t} \lambda-\lambda\right|_{L^{2}(\Sigma)}\right),
$$

which entails

$$
\begin{array}{r}
\frac{1}{\sqrt{t}}\left|u_{N}^{t}-u_{N}\right|_{H^{1}(\Omega)} \\
\leq C\left(\left|\frac{1}{\sqrt{t}}\left(A_{t}-I\right)\right|_{L^{2}(\Omega)}\left|\nabla u_{N}^{t}\right|_{L^{2}(\Omega)}\right. \\
\left.+|\lambda||\Sigma|^{1 / 2}\left|\frac{1}{\sqrt{t}}\left(w_{t}-1\right)\right|_{\infty}\right) \\
=\sqrt{t} C\left(\left|\frac{1}{t}\left(A_{t}-I\right)\right|_{L^{2}(\Omega)}\left|\nabla u_{N}^{t}\right|_{L^{2}(\Omega)}\right. \\
\left.+|\lambda||\Sigma|^{1 / 2}\left|\frac{1}{t}\left(w_{t}-1\right)\right|_{\infty}\right) .
\end{array}
$$

We now show that $\nabla u_{N}^{t}$ is bounded uniformly in $t$ in $L^{2}(\Omega)$. Since $u_{N}^{t}-1 \in H_{\Gamma, 0}^{1}(\Omega)$, we have

$$
\begin{aligned}
\alpha\left|\nabla u_{N}^{t}\right|_{L^{2}(\Omega)}^{2} & =\alpha\left|\nabla\left(u_{N}^{t}-1\right)\right|_{L^{2}(\Omega)}^{2} \\
& \leq \int_{\Omega} A_{t} \nabla\left(u_{N}^{t}-1\right) \cdot \nabla\left(u_{N}^{t}-1\right) \\
& =\int_{\Omega} A_{t} \nabla u_{N}^{t} \cdot \nabla\left(u_{N}^{t}-1\right) \\
& =|\lambda| \int_{\Sigma} w_{t}\left(u_{N}^{t}-1\right) \\
& \leq c|\lambda|\left|w_{t}\right|_{L^{\infty}}|\Sigma|^{1 / 2}|\gamma|\left|\nabla u_{N}^{t}\right|_{L^{2}(\Omega)} .
\end{aligned}
$$

Consequently,

$$
\left|\nabla u_{N}^{t}\right|_{L^{2}(\Omega)} \leq \frac{|\lambda|}{\alpha} c\left|w_{t}\right|_{L^{\infty}}|\Sigma|^{1 / 2}|\gamma|
$$

and this shows that $\nabla u_{N}^{t}$ is uniformly bounded in $L^{2}(\Omega)$ because $\left|w_{t}\right|_{\infty}$ is bounded. In addition, $A_{t}$ and $w_{t}$ are differentiable at $t=0$ by Lemma 11. Therefore,

$$
\lim _{t \rightarrow 0} \frac{1}{t}\left|u_{N}^{t}-u_{N}\right|_{H^{1}(\Omega)}^{2}=0
$$

4.4. Higher Regularity of the Solutions. In this section we will show that the solutions to the PDEs (4) and (6) have higher regularity. We begin by considering the state variable $u_{D}, u_{N} \in H^{1}(\Omega)$. For $C^{1,1}$ domains, we show that these solutions also exist in $H^{2}(\Omega)$ and more generally in $H^{k+2}(\Omega)$ if domains are of class $C^{k+1,1}, k \geq 0$.

To prove higher regularity of $u_{D}$, we require the following two theorems, which are proven in [22].

Theorem 24 (see [22, page 124]). Let $\Omega$ be a bounded open subset of $\mathbb{R}^{n}$ with a $C^{1,1}$ boundary. Consider the Dirichlet boundary value problem:

$$
\begin{aligned}
& A u=f \quad \text { in } \Omega, \\
& u=g \quad \text { on } \partial \Omega,
\end{aligned}
$$


where

$$
A=\sum_{i, j=1}^{n} D_{i}\left(a_{i j} D_{j} u\right)+\sum_{i=1}^{n} a_{i} D_{i} u+a_{0} u .
$$

Let $a_{i, j}$ be uniformly Lipschitz functions and let $a_{i}$ be bounded measurable functions such that $a_{j, i}=a_{i, j}, 1 \leq i, j \leq n$ and that there exists $\alpha>0$ with

$$
\sum_{i, j=1}^{n} a_{i, j}(x) \zeta_{i} \zeta_{j} \leq-\alpha|\zeta|^{2}
$$

for all $\zeta \in \mathbb{R}^{n}$ and for almost every $x \in \bar{\Omega}$. Assume in addition that either

(i) $a_{i}=0,1 \leq i \leq n$ and $a_{0} \geq 0$ a.e. or

(ii) $a_{0} \geq \beta>0$ a.e.

Then for every $f \in L^{p}(\Omega)$ and every $g \in W^{2-1 / p, p}(\partial \Omega)$, there exists a unique $u \in W^{2, p}(\Omega)$ that solves (110).

Theorem 25 (see [22, page 128]). Let $\Omega$ be a bounded open subset of $\mathbb{R}^{n}$ with a $C^{k+1,1}$ boundary. Consider the operator $A$ defined by (110) with $a_{i j}=a_{j i} \in C^{k, 1}(\bar{\Omega})$, and assume that there exists $\alpha>0$ such that (112) holds for all $\zeta \in \mathbb{R}^{n}$ and for every $x \in \bar{\Omega}$. Also, consider a real boundary operator $B$ which is either the identity operator (order $d=0$ ) or $B u=$ $\sum_{i=1}^{n} b_{i} D_{i} u$ with $b_{i} \in C^{k, 1}(\bar{\Omega}), 1 \leq i \leq n($ orderd $=1)$ and $\sum_{i=1}^{n} b_{i} \mathbf{n}^{i} \neq 0\left(\mathbf{n}^{i}\right.$ is the ith component of $\left.\mathbf{n}\right)$ everywhere on $\Gamma:=\partial \Omega$. Furthermore, assume that $u \in W^{2, p}(\Omega)$ satisfies $A u=f \in W^{k, p}(\Omega)$ and $\gamma B u=g \in W^{2+k-d-1 / p, p}(\Gamma)$. Then $u \in W^{k+2, p}(\Omega)$.

We will also justify the higher regularity of $u_{N}$. We use the following results whose proofs are given in the corresponding texts.

Theorem 26 (see [23, page 316]). Let $\Omega$ be a bounded open subset of $\mathbb{R}^{n}$. Suppose $u \in H^{1}(\Omega)$ is a weak solution of the PDE

$$
L u=f \quad \text { in } \Omega \text {, }
$$

where

$$
L u=-\sum_{i, j=1}^{n}\left(a^{i j}(x) u_{x_{i}}\right)_{x_{j}}+\sum_{i=1}^{n} b^{i}(x) u_{x_{i}}+c(x) u .
$$

Assume furthermore that $a^{i j}, b^{i}, c \in C^{\infty}(\Omega)$ for $i, j=$ $1,2, \ldots, n$, and $f \in C^{\infty}(\Omega)$. Then $u \in C^{\infty}(\Omega)$.

Theorem 27 (see [24, page 12]). Let $\Omega \subset \mathbb{R}^{n}$ be a bounded domain with $C^{k+1,1}$ boundary $\partial \Omega$ for some nonnegative integer $k$. Suppose the data $f$ and $g$ of the problem

$$
\begin{gathered}
-\Delta u=f \quad \text { in } \Omega, \\
u=g \quad \text { on } \partial \Omega
\end{gathered}
$$

are in $W^{k, p}(\Omega)$ and $W^{k+2-1 / p, p}(\partial \Omega)$, respectively, for some real number $p$ with $1<p<\infty$. Then $u \in W^{k+2, p}(\Omega)$.

For proof, see [22].
Theorem 28 (see [22, page 84]). Let $\Omega \subset \mathbb{R}^{n}$ be a bounded domain with $C^{1,1}$ boundary $\Gamma$ and $1<p<\infty$. Consider the Neumann problem

$$
\begin{gathered}
-\Delta u+a_{0} u=f \quad \text { in } \Omega, \\
\gamma \frac{\partial u}{\partial \mathbf{n}}=g \quad \text { on } \Gamma .
\end{gathered}
$$

If $0<a_{0} \in L^{\infty}(\Omega), f \in L^{p}(\Omega)$, and $g \in W^{1-1 / p, p}(\partial \Omega)$, then the weak solution $u$ to (116) exists in $W^{2, p}(\Omega)$.

For proof, see [22].

Using the theorems presented above, we will now prove our claim that the solutions to the PDEs (4) and (6) have indeed higher regularity. This result is given in the following theorem.

Theorem 29. Let $\Omega$ be a bounded domain with boundary of class $C^{1,1}$. Let $u_{D}, u_{N} \in H^{1}(\Omega)$ be weak solutions of the BVPs (4) and (6), respectively. Then $u_{D}$ and $u_{N}$ also belong to $H^{2}(\Omega)$. More generally, if $\Omega$ is of class $C^{k+1,1}$, where $k$ is a nonnegative integer, then $u_{D}$ and $u_{N}$ are elements of $H^{k+2}(\Omega)$.

Proof. We first consider the solution $u_{D} \in H^{1}(\Omega)$ to the Dirichlet problem (4). We use Theorem 25 to show that $u_{D}$ is an element of $H^{2}(\Omega)$. Here, (110) is applied with the following settings.

We consider $n=2$. The domain $\Omega$ is of class $C^{1,1}$. $L=-\Delta$, and hence $a_{i j}=a_{j i}=-1$ for $i=j$ and $a_{i j}=$ $a_{j i}=0$ for $i \neq j$, with $i, j=1,2$. We also observe that $\sum_{i, j=1}^{2} a_{i j}(x) \zeta_{i} \zeta_{j}=-\left(\zeta_{1}\right)^{2}-\left(\zeta_{2}\right)^{2}=-1|\zeta|^{2}$, for all $\zeta=\left(\zeta_{1}, \zeta_{2}\right)^{T}$. Thus $\alpha=1$. Furthermore, we have the following data: $f=$ $0 \in L^{2}(\Omega), g=1 \in H^{3 / 2}(\Gamma), g=0 \in H^{3 / 2}(\Sigma)$. Therefore, by using Theorem 24 , there exists a unique $u=u_{D} \in H^{2}(\Omega)$, which is a solution to (4).

For higher regularity of $u_{D}$ we apply Theorem 25 . At first we consider $C^{2,1}$-domains. In this case, $k=1$. We have $a_{i j}=$ $a_{j i}=-1$ for $i=j$ and $a_{i j}=a_{j i}=0$ for $i \neq j, i, j=1,2$. The operator $B$ is the identity operator, thus of order $d=0$. From the first consequence, it is known that $u \in H^{2}(\Omega)$ satisfies $-\Delta u=0$ and $u=g$ on $\partial \Omega$, where $g=1 \in H^{5 / 2}(\Gamma), g=$ $0 \in H^{5 / 2}(\Sigma)$. Therefore, by applying Theorem 25 , we have $u=$ $u_{D} \in H^{3}(\Omega)$. In general, for smoother domains with $C^{k+1,1}$ boundaries, solutions to (4) are elements of $H^{k+2}$.

Next, we recall that, for $C^{1,1}$ domain, there is a weak solution $u_{N} \in H^{1}(\Omega)$ to the boundary value problem (6). We also show that the solution actually lies in $H^{2}(\Omega)$ and if the domain is more regular, then so is the solution. More precisely, we want to show that if $\Omega$ is a domain whose boundary is of class $C^{k+1,1}$, then $u_{N}$ is in $H^{k+2}(\Omega)$, where $k$ is a nonnegative integer. For this purpose we need Theorem 26 which implies $u_{N} \in C^{\infty}(\Omega)$.

Choose a bounded connected domain $G$ with $C^{\infty}$ boundary $\Gamma_{1}$ such that $\bar{A} \subset G$ and $\bar{G} \subset B$, where $A$ and $B$ are the domains described in Section 2. Let $\Omega_{1}$ be the annulus having boundaries $\Gamma$ and $\Gamma_{1}$, and let $\Omega_{2}$ be the other annulus with 
boundaries $\Gamma_{1}$ and $\Sigma$. First, we consider the following elliptic problem on $\Omega_{1}$ :

$$
\begin{array}{cc}
-\Delta w=0 & \text { in } \Omega_{1}, \\
w=1 & \text { on } \Gamma, \\
w=u_{N} & \text { on } \Gamma_{1} .
\end{array}
$$

Since $\Omega_{1}$ is bounded with compact boundaries, we have $C^{\infty}\left(\Gamma \cup \Gamma_{1}\right) \subset H^{k+3 / 2}\left(\Gamma \cup \Gamma_{1}\right)$. So by applying Theorem 27, we get $w \in H^{k+2}\left(\Omega_{1}\right)$. Since $\left.u_{N}\right|_{\Omega_{1}}$ also solves (117), then by uniqueness we have $w=\left.u_{N}\right|_{\Omega_{1}}$. If $A$ is a domain with $C^{1,1}$ boundary $\Gamma$, then by Theorem 27 we have $\left.u_{N}\right|_{\Omega_{1}} \in H^{2}\left(\Omega_{1}\right)$. Moreover, if $A$ is a $C^{k+1,1}$-domain, then $\left.u_{N}\right|_{\Omega_{1}}$ is in $H^{k+2}\left(\Omega_{1}\right)$. lem:

Second, we consider the following boundary value prob-

$$
\begin{gathered}
-\Delta v+v=u_{N} \quad \text { in } \Omega_{2}, \\
\frac{\partial v}{\partial \mathbf{n}}=\frac{\partial u_{N}}{\partial \mathbf{n}} \quad \text { on } \Gamma_{1}, \\
\frac{\partial v}{\partial \mathbf{n}}=\lambda \quad \text { on } \Sigma .
\end{gathered}
$$

Because $\left.u_{N}\right|_{\Omega_{2}} \in H^{1}\left(\Omega_{2}\right)$, it follows that $\left.u_{N}\right|_{\Omega_{2}} \in L^{2}\left(\Omega_{2}\right)$. We have also shown that $u_{N} \in C^{\infty}(\Omega)$. This implies that $u_{N} \in$ $C^{\infty}\left(\Gamma_{1}\right)$. Hence, $\left(\partial u_{N} / \partial \mathbf{n}\right) \in H^{1 / 2}\left(\Gamma_{1}\right)$. Also, $\lambda \in H^{1 / 2}(\Sigma)$. Since $B$ is a domain of class $C^{1,1}$, then by Theorem 28 , we infer that (118) has a unique solution $v \in H^{2}\left(\Omega_{2}\right)$. Note, however, that $\left.u_{N}\right|_{\Omega_{2}}$ also solves (118). So by uniqueness of the solution, we get $\left.u_{N}\right|_{\Omega_{2}}=v$. Therefore, $\left.u_{N}\right|_{\Omega_{2}} \in H^{2}\left(\Omega_{2}\right)$. Now, if domain $B$ is of class $C^{k+1,1}$ and $\left.u_{N}\right|_{\Omega_{2}} \in H^{2}\left(\Omega_{2}\right) \subset H^{1}\left(\Omega_{2}\right)$, we get $v \in H^{3}\left(\Omega_{2}\right)$ by applying Theorem 25 and so is $\left.u_{N}\right|_{\Omega_{2}}$. Doing this recursively, we end up with $\left.u_{N}\right|_{\Omega_{2}} \in H^{k+2}(\Omega)$.

Hence, for $C^{k+1,1}$-domains $\Omega(:=B \backslash \bar{A})$, if we combine $\left.u_{N}\right|_{\Omega_{1}} \in H^{k+2}\left(\Omega_{1}\right)$ and $\left.u_{N}\right|_{\Omega_{2}} \in H^{k+2}\left(\Omega_{2}\right)$, we get $u_{N} \in$ $H^{k+2}\left(\Omega_{1} \cup \Omega_{2}\right)$. Moreover, $u_{N}$ is $C^{\infty}$ in a neighborhood of $\Gamma_{1}$ because $u_{N} \in C^{\infty}(\Omega)$. Therefore, $u_{N} \in H^{k+2}(\Omega)$.

Remark 30. In the computation of the first-order shape derivative, since we are dealing with $C^{1,1}$-domains, we may consider $H^{2}$-regularity for the solutions $u_{D}$ and $u_{N}$, as justified by Theorem 29.

4.5. The Shape Derivative of J. First, we state and prove the following lemma.

Lemma 31. Let $\Omega \subset \mathbb{R}^{n}$ be a bounded Lipschitz domain. Then the following equation:

$$
-\int_{\Omega}(\operatorname{div} \mathbf{F}) g d x=\int_{\Omega} \mathbf{F} \cdot \nabla g d x-\int_{\partial \Omega} g(\mathbf{F} \cdot \mathbf{n}) d s
$$

is valid for vector field $\mathbf{F}$ and scalar function $g$ having the following regularity:

(i) $\mathbf{F} \in H^{1}\left(\Omega ; \mathbb{R}^{n}\right)$ and $g \in H^{1}(\Omega)$;

(ii) $\mathbf{F} \in C^{1}\left(\bar{\Omega} ; \mathbb{R}^{n}\right)$ and $g \in W^{1,1}(\Omega)$.
Proof. First we recall the Gauss' divergence theorem in $\mathbb{R}^{n}$ saying that if a domain $\Omega \subset \mathbb{R}^{n}$ is a bounded Lipschitz domain, then we have

$$
\int_{\Omega} \operatorname{div} \mathbf{F} d x=\int_{\partial \Omega} \mathbf{F} \cdot \mathbf{n} d s
$$

for a vector field $\mathbf{F} \in C^{1}\left(\bar{\Omega}, \mathbb{R}^{n}\right)$. Second we take the divergence of the product of a scalar function $g$ and the vector field F to get

$$
\operatorname{div}(g \mathbf{F})=g \operatorname{div} \mathbf{F}+\mathbf{F} \cdot \nabla g .
$$

Then, integrating both sides over $\Omega$ and applying the divergence theorem to the vector field $g \mathbf{F}$ we obtain (119).

(i) If $\mathbf{F} \in H^{1}\left(\Omega ; \mathbb{R}^{n}\right)$ and $g \in H^{1}(\Omega)$, then $(\operatorname{div} \mathbf{F}) g \in$ $L^{1}(\Omega), \mathbf{F} \cdot \nabla g \in L^{1}(\Omega)$, and the integral $\int_{\partial \Omega} g(\mathbf{F} \cdot \mathbf{n})$ is bounded. Hence, (119) is well defined. Note that the formula

$$
-\int_{\Omega}(\operatorname{div} \mathbf{F}) g d x=\int_{\Omega} \mathbf{F} \cdot \nabla g d x-\int_{\partial \Omega} g(\mathbf{F} \cdot \mathbf{n}) d s
$$

holds for $\mathbf{F} \in C^{1}\left(\bar{\Omega} ; \mathbb{R}^{n}\right)$ and $g \in C^{1}(\bar{\Omega})$. We write

$$
-\int_{\Omega}(\operatorname{div} \mathbf{F}) g d x=\int_{\Omega} \mathbf{F} \cdot \nabla g d x-\int_{\partial \Omega} \gamma_{0} g\left(\gamma_{0} \mathbf{F} \cdot \mathbf{n}\right) d s,
$$

where $\gamma_{0}: H^{1}(\Omega) \rightarrow L^{2}(\partial \Omega)$ is a trace operator. Let $\mathbf{F} \epsilon$ $H^{1}\left(\Omega ; \mathbb{R}^{n}\right)$ and $g \in H^{1}(\Omega)$. By density, we pick $\left\{\mathbf{F}_{k}\right\} \subset C^{1}(\bar{\Omega}$ and $\left\{g_{k}\right\} \subset C^{1}(\bar{\Omega})$ such that $\mathbf{F}_{k} \rightarrow \mathbf{F}$ in $H^{1}\left(\bar{\Omega} ; \mathbb{R}^{n}\right)$ and $g_{k} \rightarrow$ $g$ in $H^{1}(\Omega)$. By (122),

$$
-\int_{\Omega}\left(\operatorname{div} \mathbf{F}_{k}\right) g_{k} d x=\int_{\Omega} \mathbf{F}_{k} \cdot \nabla g_{k} d x-\int_{\partial \Omega} g_{k}\left(\mathbf{F}_{k} \cdot \mathbf{n}\right) d s .
$$

Note that $\gamma_{0} \mathbf{F}_{k}=\left.\mathbf{F}_{k}\right|_{\partial \Omega}$ and $\gamma_{0} g_{k}=\left.g_{k}\right|_{\partial \Omega}$. Also $\mathbf{F}_{k} \rightarrow \mathbf{F}$ in $H^{1}\left(\bar{\Omega} ; \mathbb{R}^{n}\right)$ implies that $\operatorname{div} \mathbf{F}_{k} \rightarrow \operatorname{div} \mathbf{F}$ in $L^{2}(\Omega)$. Moreover, $g_{k} \rightarrow g$ in $H^{1}(\Omega)$ implies $\nabla g_{k} \rightarrow \nabla g$ in $L^{2}(\Omega)$. Furthermore, since $\gamma_{0} \in \mathscr{L}\left(H^{1}(\Omega), L^{2}(\partial \Omega)\right), \gamma_{0} \mathbf{F}_{k} \rightarrow \gamma_{0} \mathbf{F}$ and $\gamma_{0} g_{k} \rightarrow \gamma_{0} g$ in $L^{2}(\partial \Omega)$. Therefore, (119) holds for $\mathbf{F} \in$ $H^{1}\left(\Omega ; \mathbb{R}^{n}\right)$ and $g \in H^{1}(\Omega)$.

(ii) If $\mathbf{F} \in C^{1}\left(\bar{\Omega} ; \mathbb{R}^{n}\right)$ and $g \in W^{1,1}(\Omega)$, then $(\operatorname{div} \mathbf{F}) g \in$ $L^{1}(\Omega)$ and $\mathbf{F} \cdot \nabla g \in L^{1}(\Omega)$. Note that $\gamma g \in L^{1}(\partial \Omega)$ (cf. [25, page 316]), where $\gamma g$ refers to the trace of $g$ on $\partial \Omega$, hence $g(\mathbf{F}$. n) $\in L^{1}(\partial \Omega)$. Therefore, (119) is also well defined for this case. Using similar arguments as above and using the density of $C^{1}(\bar{\Omega})$ in $W^{1,1}(\Omega)$ we can show that (119) is also valid for this case.

Now we apply Lemma 31 to prove the next lemma.

Lemma 32 (see [1]). Let $u_{D}$ and $u_{N}$ belonging to $H^{2}(\Omega)$ satisfy the Dirichlet problem (4) and the Neumann problem (6), respectively. Then

$$
\begin{gathered}
\int_{\Omega} A \nabla u_{D} \cdot \nabla u_{D}=-\int_{\Sigma}\left(\nabla u_{D} \cdot \mathbf{n}\right)^{2} \mathbf{V} \cdot \mathbf{n}, \\
\int_{\Omega} A \nabla u_{N} \cdot \nabla u_{N}=\int_{\Sigma}\left|\nabla u_{N}\right|^{2} \mathbf{V} \cdot \mathbf{n}-2 \lambda \int_{\Sigma} \mathbf{V} \cdot \nabla u_{N},
\end{gathered}
$$

where $A$ is given by property (12) of Lemma 11. 
Proof. From Lemma 11, we recall the expression for $A$, which is given by $A=(\operatorname{div} \mathbf{V}) I-\left((D \mathbf{V})+(D \mathbf{V})^{T}\right)$. Our first goal is to derive an expression for $\int_{\Omega} A \nabla u \cdot \nabla v$ for $u, v \in H^{2}(\Omega)$. We begin by writing $\int_{\Omega} A \nabla u \cdot \nabla v$ as follows:

$$
\begin{aligned}
\int_{\Omega} A \nabla u \cdot \nabla v= & \int_{\Omega}(\operatorname{div} \mathbf{V}) \nabla u \cdot \nabla v-\int_{\Omega}(D \mathbf{V}) \nabla u \cdot \nabla v \\
& -\int_{\Omega}(D \mathbf{V})^{T} \nabla u \cdot \nabla v .
\end{aligned}
$$

We manipulate each term on the right-hand side of (127). First, because $u, v \in H^{2}(\Omega)$, we have $\nabla u \cdot \nabla v \in W^{1,1}(\Omega)$. Hence we can use (119) by taking $g=\nabla u \cdot \nabla v$ and by choosing $\mathbf{F}=\mathbf{V}$. In addition, we take into account that $\mathbf{V}$ vanishes on the fixed boundary $\Gamma$. This leads to

$$
\begin{aligned}
-\int_{\Omega}(\operatorname{div} \mathbf{V}) \nabla u \cdot \nabla v & =\int_{\Omega} \nabla(\nabla u \cdot \nabla v) \cdot \mathbf{V} \\
& -\int_{\Sigma}(\nabla u \cdot \nabla v) \mathbf{V} \cdot \mathbf{n} .
\end{aligned}
$$

The other two terms on the right hand side of (127) are manipulated as follows. The term $\nabla(\nabla u \cdot \nabla v) \cdot \mathbf{V}$ is written as

$$
\nabla(\nabla u \cdot \nabla v) \cdot \mathbf{V}=(\nabla v)^{T}\left(\left(\nabla^{2} u\right) \mathbf{V}\right)+\mathbf{V}^{T}\left(\left(\nabla^{2} v\right) \nabla u\right)
$$

where $\nabla^{2} u$ represents the Hessian of $u$. Because Hessian is symmetric, we obtain

$$
\nabla(\nabla u \cdot \nabla v) \cdot \mathbf{V}=\left(\nabla^{2} u \nabla v+\nabla^{2} v \nabla u\right) \cdot \mathbf{V} .
$$

Substituting (130) into (128) we get

$$
\begin{aligned}
\int_{\Omega} & (\operatorname{div} \mathbf{V}) \nabla u \cdot \nabla v \\
= & -\int_{\Omega}\left(\left(\nabla^{2} u\right) \nabla v+\left(\nabla^{2} v\right) \nabla u\right) \cdot \mathbf{V} \\
& +\int_{\Sigma}(\nabla u \cdot \nabla v) \mathbf{V} \cdot \mathbf{n} .
\end{aligned}
$$

Next, we expand the expression $\operatorname{div}((\mathbf{V} \cdot \nabla u) \nabla v)$ using (121) as

$$
\begin{aligned}
\operatorname{div}((\mathbf{V} \cdot \nabla u) \nabla v)= & (\mathbf{V} \cdot \nabla u) \Delta v+\nabla v \cdot(\mathbf{V} \cdot \nabla u) \\
= & (\mathbf{V} \cdot \nabla u) \Delta v+\nabla v \cdot\left[(D \mathbf{V})^{T} \nabla u+\nabla^{2} u \mathbf{V}\right] \\
= & (D \mathbf{V}) \nabla v \cdot \nabla u \\
& +(\nabla v)^{T}\left(\nabla^{2} u\right) \mathbf{V}+(\mathbf{V} \cdot \nabla u) \Delta v
\end{aligned}
$$

Integrating both sides of (132) over $\Omega$, applying Stoke's theorem, and considering $\mathbf{V}=0$ on $\Gamma$ we end up with

$$
\begin{aligned}
\int_{\Omega} & (D \mathbf{V}) \nabla v \cdot \nabla u+\int_{\Omega}(\nabla v)^{T}\left(\nabla^{2} u\right) \mathbf{V}+\int_{\Omega}(\mathbf{V} \cdot \nabla u) \Delta v \\
\quad= & \int_{\Sigma}(\mathbf{V} \cdot \nabla u) \nabla v \cdot \mathbf{n},
\end{aligned}
$$

or equivalently

$$
\begin{aligned}
& \int_{\Omega}(D \mathbf{V}) \nabla v \cdot \nabla u \\
& \quad=-\int_{\Omega}(\Delta v)(\mathbf{V} \cdot \nabla u)-\int_{\Omega}\left(\nabla^{2} u\right) \nabla v \cdot \mathbf{V}+\int_{\Sigma} \frac{\partial v}{\partial \mathbf{n}}(\mathbf{V} \cdot \nabla u) .
\end{aligned}
$$

Interchanging $v$ and $u$ we get

$$
\begin{aligned}
& \int_{\Omega}(D \mathbf{V}) \nabla u \cdot \nabla v \\
& \quad=-\int_{\Omega}(\Delta u) \mathbf{V} \cdot \nabla v-\int_{\Omega}\left(\nabla^{2} v\right) \nabla u \cdot \mathbf{V}+\int_{\Sigma} \frac{\partial u}{\partial \mathbf{n}}(\mathbf{V} \cdot \nabla v) .
\end{aligned}
$$

Also, because $(D \mathbf{V})^{T} \nabla v \cdot \nabla u=(D \mathbf{V}) \nabla u \cdot \nabla v$, we obtain

$$
\begin{aligned}
& \int_{\Omega}(D \mathbf{V})^{T} \nabla v \cdot \nabla u \\
& \quad=-\int_{\Omega}(\Delta u) \mathbf{V} \cdot \nabla v-\int_{\Omega}\left(\nabla^{2} v\right) \nabla u \cdot \mathbf{V}+\int_{\Sigma} \frac{\partial u}{\partial \mathbf{n}}(\mathbf{V} \cdot \nabla v) .
\end{aligned}
$$

Thus,

$$
\begin{aligned}
\int_{\Omega}(D \mathbf{V})^{T} \nabla u \cdot \nabla v \\
\quad=-\int_{\Omega}(\Delta v) \mathbf{V} \cdot \nabla u-\int_{\Omega}\left(\nabla^{2} u\right) \nabla v \cdot \mathbf{V}+\int_{\Sigma} \frac{\partial v}{\partial \mathbf{n}}(\mathbf{V} \cdot \nabla u) .
\end{aligned}
$$

Adding (131), (135), and (137) altogether, we express (127) as

$$
\begin{aligned}
\int_{\Omega} A \nabla u \cdot \nabla v= & \int_{\Omega} \Delta u(\mathbf{V} \cdot \nabla v) \\
& +\int_{\Omega} \Delta v(\mathbf{V} \cdot \nabla u)-\int_{\Sigma} \frac{\partial v}{\partial \mathbf{n}}(\mathbf{V} \cdot \nabla u) \\
& -\int_{\Sigma} \frac{\partial u}{\partial \mathbf{n}}(\mathbf{V} \cdot \nabla v)+\int_{\Sigma}(\nabla u \cdot \nabla v) \mathbf{V} \cdot \mathbf{n} .
\end{aligned}
$$

Set $u=v=u_{D}$ in (138). The first two integrals on the right hand side of (138) vanish because $-\Delta u_{D}=0$ in $\Omega$. Moreover, since $u_{D}=0$ on $\Sigma$ we have $\nabla u_{D}=\left(\partial u_{D} / \partial \mathbf{n}\right) \mathbf{n}$. Thus, we can write (138) as follows:

$$
\begin{aligned}
\int_{\Omega} & A \nabla u_{D} \cdot \nabla u_{D} \\
= & -2 \int_{\Sigma}\left(\nabla u_{D} \cdot \mathbf{n}\right)\left(\mathbf{V} \cdot \nabla u_{D}\right)+\int_{\Sigma}\left(\nabla u_{D} \cdot \nabla u_{D}\right) \mathbf{V} \cdot \mathbf{n} \\
= & -2 \int_{\Sigma}\left(\frac{\partial u_{D}}{\partial \mathbf{n}}\right)\left(\frac{\partial u_{D}}{\partial \mathbf{n}} \mathbf{V} \cdot \mathbf{n}\right)+\int_{\Sigma}\left(\frac{\partial u_{D}}{\partial \mathbf{n}}\right)^{2} \mathbf{V} \cdot \mathbf{n} \\
= & -\int_{\Sigma}\left(\nabla u_{D} \cdot \mathbf{n}\right)^{2} \mathbf{V} \cdot \mathbf{n} .
\end{aligned}
$$

Therefore, (125) is satisfied. 
On the other hand, by replacing both $u$ and $v$ by $u_{N}$ and by considering that $-\Delta u_{N}=0$ in $\Omega$ and $\partial u_{N} / \partial \mathbf{n}=\lambda$ on $\Sigma$, we derive (126) as

$$
\begin{aligned}
\int_{\Omega} & A \nabla u_{N} \cdot \nabla u_{N} \\
= & -2 \int_{\Sigma} \frac{\partial u_{N}}{\partial \mathbf{n}}\left(\mathbf{V} \cdot \nabla u_{N}\right)+\int_{\Sigma}\left(\nabla u_{N} \cdot \nabla u_{N}\right) \mathbf{V} \cdot \mathbf{n} \\
= & \int_{\Sigma}\left|\nabla u_{N}\right|^{2} \mathbf{V} \cdot \mathbf{n}-2 \lambda \int_{\Sigma} \mathbf{V} \cdot \nabla u_{N} .
\end{aligned}
$$

Now, we derive the explicit form of the first-order shape derivative of $J$.

Theorem 33. For $C^{1,1}$ bounded domain $\Omega$, the first-order shape derivative of the Kohn-Vogelius cost functional

$$
J(\Omega)=\frac{1}{2} \int_{\Omega}\left|\nabla\left(u_{D}-u_{N}\right)\right|^{2} d x
$$

in the direction of a perturbation field $\mathbf{V} \in \Theta$, where $\Theta$ is defined by (13) and the state functions $u_{D}$ and $u_{N}$ satisfy the Dirichlet problem (4) and the Neumann problem (6), respectively, is given by

$$
\begin{aligned}
d J(\Omega ; \mathbf{V})=\frac{1}{2} \int_{\Sigma} & \left(\lambda^{2}-\left(\nabla u_{D} \cdot \mathbf{n}\right)^{2}\right. \\
& \left.+2 \lambda \kappa u_{N}-\left(\nabla u_{N} \cdot \tau\right)^{2}\right) \mathbf{V} \cdot \mathbf{n} d s,
\end{aligned}
$$

where $\mathbf{n}$ is the unit exterior normal vector to $\Sigma, \tau$ is a unit tangent vector to $\Sigma$, and $\kappa$ is the mean curvature of $\Sigma$.

Proof. First we consider the functionals defined on the reference domain and perturbed domains

$$
\begin{gathered}
J(\Omega)=\frac{1}{2} \int_{\Omega}\left|\nabla\left(u_{D}-u_{N}\right)\right|^{2} d x, \\
J\left(\Omega_{t}\right)=\frac{1}{2} \int_{\Omega_{t}}\left|\nabla\left(u_{D, t}-u_{N, t}\right)\right|^{2} d x_{t} .
\end{gathered}
$$

Let $z_{t}=u_{D, t}-u_{N, t}$ and $z=u_{D}-u_{N}$. Note that if $z^{t}=z_{t} \circ T_{t}$, then we have $D z^{t}=\left(D z_{t} \circ T_{t}\right)\left(D T_{t}\right)$. Hence

$$
M_{t} \nabla z^{t}=\left(\nabla z_{t}\right) \circ T_{t} .
$$

By this together with Lemma 16, we can write $J\left(\Omega_{t}\right)$ as follows:

$$
\begin{aligned}
J\left(\Omega_{t}\right) & =\frac{1}{2} \int_{\Omega_{t}}\left|\nabla z_{t}\right|^{2} d x_{t}=\frac{1}{2} \int_{\Omega} I_{t}\left|\left(\nabla z_{t}\right) \circ T_{t}\right|^{2} d x \\
& =\frac{1}{2} \int_{\Omega} I_{t}\left|M_{t} \nabla z^{t}\right|^{2} d x=\frac{1}{2} \int_{\Omega}\left\langle\nabla z^{t}, A_{t} \nabla z^{t}\right\rangle .
\end{aligned}
$$

Then we write $J\left(\Omega_{t}\right)-J(\Omega)$ as

$$
\begin{aligned}
J\left(\Omega_{t}\right)-J(\Omega)= & \frac{1}{2} \int_{\Omega}\left(\left\langle\nabla z^{t}, A_{t} \nabla z^{t}\right\rangle-\langle\nabla z, \nabla z\rangle\right) d x \\
= & \frac{1}{2} \int_{\Omega}\left(\left\langle\nabla z^{t},\left(A_{t}-I\right) \nabla z^{t}\right\rangle\right. \\
& \left.\quad+\left(\left|\nabla z^{t}\right|^{2}-|\nabla z|^{2}\right)\right) d x \\
=: & J_{1}(t)+J_{2}(t) .
\end{aligned}
$$

Using Lemma 11, the symmetry of $A$, and noting that $z^{t} \rightarrow z$ as $t \rightarrow 0$ we have

$$
J_{1}(t)=\frac{1}{2} \int_{\Omega}\left\langle\nabla z^{t},\left(A_{t}-I\right) \nabla z^{t}\right\rangle d x, \quad J_{1}(0)=0 .
$$

Hence,

$$
\begin{aligned}
\dot{J}_{1}(0)= & \lim _{t \rightarrow 0} \frac{J_{1}(t)-J_{1}(0)}{t} \\
= & \frac{1}{2} \lim _{t \rightarrow 0} \int_{\Omega}\left\langle\nabla z^{t}, \frac{A_{t}-I}{t} \nabla z^{t}\right\rangle d x \\
= & \frac{1}{2} \int_{\Omega}\langle\nabla z, A \nabla z\rangle d x \\
= & \frac{1}{2} \int_{\Omega}\left\langle\nabla\left(u_{D}-u_{N}\right), A \nabla\left(u_{D}-u_{N}\right)\right\rangle d x \\
= & \frac{1}{2} \int_{\Omega} A \nabla u_{D} \cdot \nabla u_{D} d x+\frac{1}{2} \int_{\Omega} A \nabla u_{N} \cdot \nabla u_{N} d x \\
& -\int_{\Omega} A \nabla u_{D} \cdot \nabla u_{N} d x .
\end{aligned}
$$

To manipulate $J_{2}(t)$ we use the identity $a^{2}-b^{2}=(a-b)^{2}+$ $2 b(a-b)$ as

$$
\begin{aligned}
J_{2}(t) & =\frac{1}{2} \int_{\Omega}\left(\left|\nabla z^{t}\right|^{2}-|\nabla z|^{2}\right) d x \\
& =\frac{1}{2} \int_{\Omega}\left|\nabla\left(z^{t}-z\right)\right|^{2} d x+\int_{\Omega}\left\langle\nabla z, \nabla\left(z^{t}-z\right)\right\rangle d x \\
& =: J_{21}(t)+J_{22}(t) .
\end{aligned}
$$

$J_{21}(t)$ is manipulated as follows:

$$
\begin{aligned}
J_{21}(t) & =\frac{1}{2} \int_{\Omega}\left|\nabla\left(z^{t}-z\right)\right|^{2} \\
& =\frac{1}{2} \int_{\Omega}\left|\nabla\left(\left(u_{D}^{t}-u_{N}^{t}\right)-\left(u_{D}-u_{N}\right)\right)\right|^{2} \\
& \leq \int_{\Omega}\left|\nabla\left(u_{D}^{t}-u_{D}\right)\right|^{2}+\int_{\Omega}\left|\nabla\left(u_{N}^{t}-u_{N}\right)\right|^{2} .
\end{aligned}
$$


Applying Theorems 22 and 23 yields $\dot{J}_{21}=0 . J_{22}(t)$ is treated as follows:

$$
\begin{aligned}
J_{22}(t)= & \int_{\Omega}\left\langle\nabla z, \nabla\left(z^{t}-z\right)\right\rangle d x \\
= & \int_{\Omega}\left\langle\nabla\left(u_{D}-u_{N}\right), \nabla\left(\left(u_{D}^{t}-u_{D}\right)-\left(u_{N}^{t}-u_{N}\right)\right)\right\rangle \\
= & \int_{\Omega}\left\langle\nabla u_{D}, \nabla\left(u_{D}^{t}-u_{D}\right)\right\rangle-\int_{\Omega}\left\langle\nabla u_{N}, \nabla\left(u_{D}^{t}-u_{D}\right)\right\rangle \\
& -\int_{\Omega}\left\langle\nabla\left(u_{D}-u_{N}\right), \nabla\left(u_{N}^{t}-u_{N}\right)\right\rangle d x .
\end{aligned}
$$

Since $u_{D}^{t}-u_{D} \in H_{0}^{1}(\Omega)$, the variational equation for $u_{D}$ and $u_{N}$ implies

$$
\begin{aligned}
J_{22}(t)= & -\int_{\Omega}\left\langle\nabla\left(u_{D}-u_{N}\right), \nabla\left(u_{N}^{t}-u_{N}\right)\right\rangle d x \\
= & \int_{\Omega}\left\langle\nabla u_{N}, \nabla\left(u_{D}-u_{N}\right)\right\rangle \\
& -\int_{\Omega}\left\langle\nabla u_{N}^{t}, \nabla\left(u_{D}-u_{N}\right)\right\rangle d x .
\end{aligned}
$$

Choosing $u_{D}-u_{N} \in H_{\Gamma, 0}^{1}(\Omega)$ as test function and applying $(65)$ and $(84), J_{22}(t)$ can be written as

$$
\begin{aligned}
J_{22}(t)= & \lambda \int_{\Sigma}\left(u_{D}-u_{N}\right)-\int_{\Omega}\left\langle A_{t} \nabla u_{N}^{t}, \nabla\left(u_{D}-u_{N}\right)\right\rangle \\
& +\int_{\Omega}\left\langle\left(A_{t}-I\right) \nabla u_{N}^{t}, \nabla\left(u_{D}-u_{N}\right)\right\rangle \\
= & \lambda \int_{\Sigma}\left(1-w_{t}\right)\left(u_{D}-u_{N}\right) d s \\
& +\int_{\Omega}\left\langle\left(A_{t}-I\right) \nabla u_{N}^{t}, \nabla\left(u_{D}-u_{N}\right)\right\rangle .
\end{aligned}
$$

From this we obtain

$$
\dot{J}_{22}(0)=\lambda \int_{\Sigma} u_{N} \operatorname{div}_{\Sigma} \mathbf{V}+\int_{\Omega}\left\langle A \nabla u_{N}, \nabla\left(u_{D}-u_{N}\right)\right\rangle,
$$

and therefore,

$$
\dot{J}_{2}(0)=\lambda \int_{\Sigma} u_{N} \operatorname{div}_{\Sigma} \mathbf{V}+\int_{\Omega}\left\langle A \nabla u_{N}, \nabla\left(u_{D}-u_{N}\right)\right\rangle .
$$

Combining $\dot{J}_{1}(0)$ and $\dot{J}_{2}(0)$ we get

$$
\begin{aligned}
d J(\Omega ; \mathbf{V}):= & \lim _{t \rightarrow 0^{+}} \frac{J\left(\Omega_{t}\right)-J(\Omega)}{t}=\dot{J}_{1}(0)+\dot{J}_{2}(0) \\
= & \frac{1}{2} \int_{\Omega} A \nabla u_{D} \cdot \nabla u_{D}+\frac{1}{2} \int_{\Omega} A \nabla u_{N} \cdot \nabla u_{N} \\
& -\int_{\Omega} A \nabla u_{D} \cdot \nabla u_{N}+\lambda \int_{\Sigma}\left(\operatorname{div}_{\Sigma} \mathbf{V}\right) u_{N}
\end{aligned}
$$

$$
\begin{aligned}
& +\int_{\Omega}\left\langle A \nabla u_{N}, \nabla\left(u_{D}-u_{N}\right)\right\rangle \\
= & \frac{1}{2} \int_{\Omega} A \nabla u_{D} \cdot \nabla u_{D}-\frac{1}{2} \int_{\Omega} A \nabla u_{N} \cdot \nabla u_{N} \\
& +\lambda \int_{\Sigma}\left(\operatorname{div}_{\Sigma} \mathbf{V}\right) u_{N} .
\end{aligned}
$$

We know from the previous section that $u_{D}$ and $u_{N}$ exist in $H^{2}(\Omega)$ since $\Omega$ is of class $C^{1,1}$. Using this smoothness we can now apply Lemma 32 and write (156) as follows:

$$
\begin{aligned}
d J(\Omega ; \mathbf{V})= & -\frac{1}{2} \int_{\Sigma}\left(\nabla u_{D} \cdot \mathbf{n}\right)^{2} \mathbf{V} \cdot \mathbf{n} \\
& -\frac{1}{2}\left[\int_{\Sigma}\left|\nabla u_{N}\right|^{2} \mathbf{V} \cdot \mathbf{n}-2 \lambda \int_{\Sigma} \mathbf{V} \cdot \nabla u_{N}\right] \\
& +\lambda \int_{\Sigma} \operatorname{div}_{\Sigma} \mathbf{V} u_{N} \\
= & -\frac{1}{2} \int_{\Sigma}\left(\nabla u_{D} \cdot \mathbf{n}\right)^{2} \mathbf{V} \cdot \mathbf{n} \\
& +\lambda \int_{\Sigma}\left(\mathbf{V} \cdot \nabla u_{N}+u_{N} \operatorname{div}_{\Sigma} \mathbf{V}\right) \\
& -\frac{1}{2} \int_{\Sigma}\left|\nabla u_{N}\right|^{2} \mathbf{V} \cdot \mathbf{n} .
\end{aligned}
$$

Since

$$
\int_{\Sigma}\left(\left(\nabla u_{N}\right) \cdot \mathbf{V}+u_{N} \operatorname{div}_{\Sigma} \mathbf{V}\right)=\int_{\Sigma}\left(\lambda+u_{N} \operatorname{div}_{\Sigma} \mathbf{n}\right) \mathbf{V} \cdot \mathbf{n}
$$

and $\left|\nabla u_{N}\right|^{2}=\lambda^{2}+\left(\nabla u_{N} \cdot \tau\right)^{2}$, we obtain the first-order shape derivative of $J$ :

$$
\begin{aligned}
d J(\Omega ; \mathbf{V})=\frac{1}{2} \int_{\Sigma}( & -\lambda^{2}-\left(\nabla u_{N} \cdot \tau\right)^{2} \\
& \left.+2 \lambda^{2}+2 \lambda u_{N} \kappa-\left(\nabla u_{D} \cdot \mathbf{n}\right)^{2}\right) \mathbf{V} \cdot \mathbf{n} .
\end{aligned}
$$

\section{Conclusion}

In this paper we derived the explicit form of the firstorder Eulerian shape derivative of the Kohn-Vogelius cost functional given by (7) in a rigorous manner. As seen in the presentation, we can avoid working on the shape derivatives of the states and apply their Hölder continuity instead. We employed techniques similar to $[9,13]$ but it was not necessary to introduce adjoint variables. For the shape derivative of the cost functional to be well defined we observe that we can consider domains with $C^{1,1}$ boundaries and we need $H^{2}$ regularity for the state variables.

Rewriting the first-order shape derivative as $d J(\Omega ; \mathbf{V})=$ $\int_{\Sigma} F \mathbf{V} \cdot \mathbf{n}$, where

$$
F=\frac{1}{2}\left(-\left(\nabla u_{N} \cdot \tau\right)^{2}-\left(\nabla u_{D} \cdot \mathbf{n}\right)^{2}+\lambda^{2}+2 \lambda u_{N} \kappa\right),
$$


we conclude that $J$ is shape differentiable at $\Omega$. This is because $d J(\Omega ; \mathbf{V})$ exists for all $\mathbf{V} \in \Theta$ and the mapping $\mathbf{V} \mapsto d J(\Omega ; \mathbf{V})$ is linear and continuous with respect to $\mathbf{V} \in \Theta$ since

$$
|d J(\Omega ; \mathbf{V})| \leq|F|_{L^{1}(\Sigma)}|\mathbf{V}|_{C(\Sigma)} \leq|F|_{L^{1}(\Sigma)}|\mathbf{V}|_{C^{1,1}(\bar{U})} .
$$

We also observe that the shape derivative of $J$ which is formulated from the Bernoulli free boundary problem depends on the normal component of the deformation field $\mathbf{V}$ at the free boundary $\Sigma$; that is, there exists a function $g_{\Omega}$ defined on the free boundary $\Sigma$ such that

$$
d J(\Omega ; \mathbf{V})=\int_{\Sigma} g_{\Omega} \mathbf{V} \cdot \mathbf{n} d s
$$

This agrees with the Hadamard structure theorem [26, 27].

Theorem 34 (see [26, page 318]). Let $\Omega$ be a domain with $C^{k+1}$ boundary $\Gamma$ for some integer $k \geq 0$. Assuming that at $\Omega$ a shape gradient of $J(\Omega)$ exists. Then there exists a scalar distribution $g$ in $\mathscr{D}^{k}(\Gamma)^{\prime}$ such that

$$
d J(\Omega ; \mathbf{V})=\langle g(\Omega), \mathbf{V} \cdot \mathbf{n}\rangle_{\mathscr{D}^{k}(\Gamma)^{\prime} \times \mathscr{D}^{k}(\Gamma)},
$$

where $\mathscr{D}^{k}(\Gamma)$ is the space of $C^{k}$ functions from $\Gamma$ to $\mathbb{R}^{n}$, and $\mathbf{V} \cdot \mathbf{n}$ is the normal component of $\mathbf{V}$ on $\Gamma$.

Proof. See [26].

\section{Acknowledgments}

The paper is partially supported by the ÖAD-Austrian Agency for International Cooperation in Education and Research for the Technologiestipendien Südostasien (Doktorat) scholarship in the frame of the ASEA-Uninet; the SFB Research Center "Mathematical Optimization and Applications in Biomedical Sciences" SFB F32; the University of the Philippines Baguio. The first author is thankful to the Institute for Mathematics and Scientific Computing, University of Graz, for funding a presentation of partial results during the 7th European Conference on Elliptic and Parabolic Problems held on May 21-25, 2012 at Gaeta, Italy. He is likewise grateful to Professor Gilbert Peralta for his helpful insights and suggestions. Moreover, he is thanking UP Baguio for giving a Ph.D. incentive grant for writing this paper. Last but not least, the authors would like to thank the referees for devoting time to review this material.

\section{References}

[1] B. Abda, F. Bouchon, G. Peichl, M. Sayeh, and R. Touzani, "A new formulation for the Bernoulli problem," in Proceedings of the 5th International Conference on Inverse Problems, Control and Shape Optimization, pp. 1-19, 2010.

[2] L. A. Caffarelli and S. Salsa, A Geometric Approach to Free Boundary Problems, American Mathematical Society, Providence, RI, USA, 2005.

[3] J. Crank, Free and Moving Boundary Problems, Oxford University Press, New York, NY, USA, 1984.
[4] M. Flucher and M. Rumpf, "Bernoulli's free-boundary problem, qualitative theory and numerical approximation," Journal fur die Reine und Angewandte Mathematik, vol. 486, pp. 165-204, 1997.

[5] A. Friedman, "Free boundary problems in science and technology," Notices of the AMS, vol. 47, pp. 854-861, 2000.

[6] J. I. Toivanen, J. Haslinger, and R. A. E. Mäkinen, "Shape optimization of systems governed by Bernoulli free boundary problems," Computer Methods in Applied Mechanics and Engineering, vol. 197, no. 45-48, pp. 3803-3815, 2008.

[7] A. Beurling, "On free boundary problems for the Laplace equation," in Proceedings of the Seminars on Analytic Functions, pp. 248-263, 1957.

[8] P. Cardaliaguet and R. Tahraoui, "Some uniqueness results for Bernoulli interior free-boundary problems in convex domains," Electronic Journal of Differential Equations, vol. 2002, pp. 1-16, 2002.

[9] J. Haslinger, K. Ito, T. Kozubek, K. Kunisch, and G. Peichl, “On the shape derivative for problems of Bernoulli type," Interfaces and Free Boundaries, vol. 11, no. 2, pp. 317-330, 2009.

[10] J. Haslinger, T. Kozubek, K. Kunisch, and G. Peichl, "Shape optimization and fictitious domain approach for solving free boundary problems of Bernoulli type," Computational Optimization and Applications, vol. 26, no. 3, pp. 231-251, 2003.

[11] K. Eppler and H. Harbrecht, "Shape optimization for free boundary problems-analysis and numerics," in Constrained Optimization and Optimal Control for Partial Differential Equations, vol. 160, pp. 277-288, 2012.

[12] K. Eppler and H. Harbrecht, "Tracking Neumann data for stationary free boundary problems," SIAM Journal on Control and Optimization, vol. 48, no. 5, pp. 2901-2916, 2009.

[13] K. Ito, K. Kunisch, and G. H. Peichl, "Variational approach to shape derivatives for a class of Bernoulli problems," Journal of Mathematical Analysis and Applications, vol. 314, no. 1, pp. 126149, 2006.

[14] R. Kohn and M. Vogelius, "Determining conductivity by boundary measurements," Communications on Pure and Applied Mathematics, vol. 37, no. 3, pp. 289-298, 1984.

[15] K. Eppler and H. Harbrecht, "On a Kohn-Vogelius like formulation of free boundary problems," Computational Optimization and Applications, vol. 52, no. 1, pp. 69-85, 2012.

[16] M. Delfour and J. Zolesio, Shapes and Geometries, SIAM, Philadelphia, Pa, USA, 2001.

[17] P. Ciarlet, Mathematical Elasticity I, Elsevier Science Publishers B.V., Amsterdam, The Netherlands, 1988.

[18] J. Sokolowski and J. Zolesio, Introduction to Shape Optimization, Springer, Berlin, Germany, 1991.

[19] J. Bacani, Methods of shape optimization in free boundary problems [Ph.D. thesis], Karl-Franzens-Universitaet Graz, Graz, Austria, 2013.

[20] J. Haslinger and R. A. E. Mäkinen, Introduction to Shape Optimization (Theory, Approximation, and Computation), SIAM Advances and Control, Philadelphia, Pa, USA, 2003.

[21] T. Tiihonen, "Shape optimization and trial methods for free boundary problems," Mathematical Modelling and Numerical Analysis, vol. 31, no. 7, pp. 805-825, 1997.

[22] P. Grisvard, Elliptic Problems in Nonsmooth Domains, Pitman Publishing, Marshfield, Mass, USA, 1985.

[23] L. C. Evans, Partial Differential Equations, American Mathematical Society, Providence, RI, USA, 1998. 
[24] V. Girault and P. Raviart, Finite Element Methods for NavierStoke's Equations (Theory and Algorithms), Springer, Berlin, Germany, 1986.

[25] A. Kufner, O. John, and S. Fucik, Function Spaces, Noordhoff International Publishing, Leyden, The Netherlands, 1977.

[26] M. C. Delfour and J. P. Zolésio, "Anatomy of the shape Hessian," Annali di Matematica Pura ed Applicata, vol. 159, no. 1, pp. 315339, 1991.

[27] J. Lamboley and M. Pierre, "Structure of shape derivatives around irregular domains and applications," Journal of Convex Analysis, vol. 14, no. 4, pp. 807-822, 2007. 


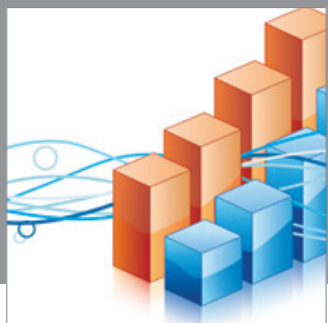

Advances in

Operations Research

mansans

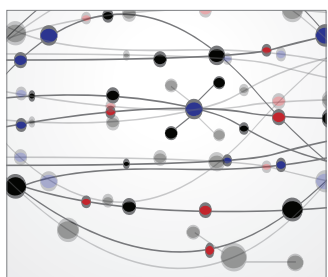

The Scientific World Journal
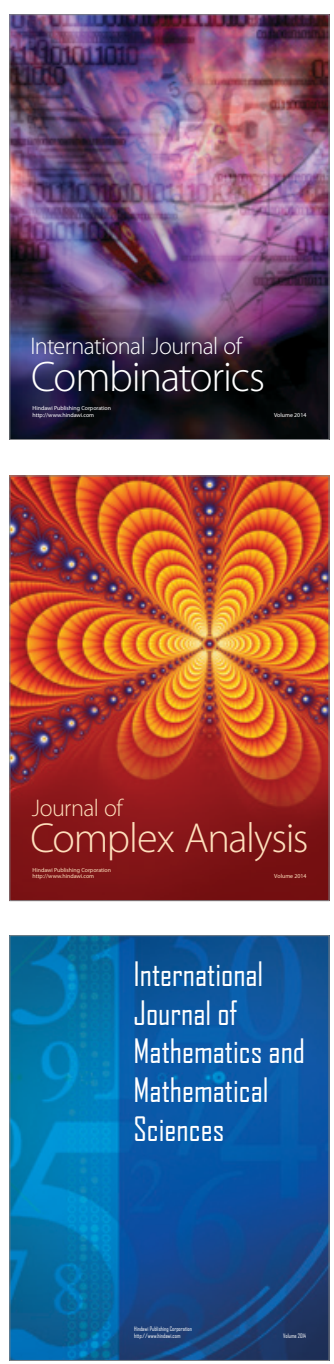
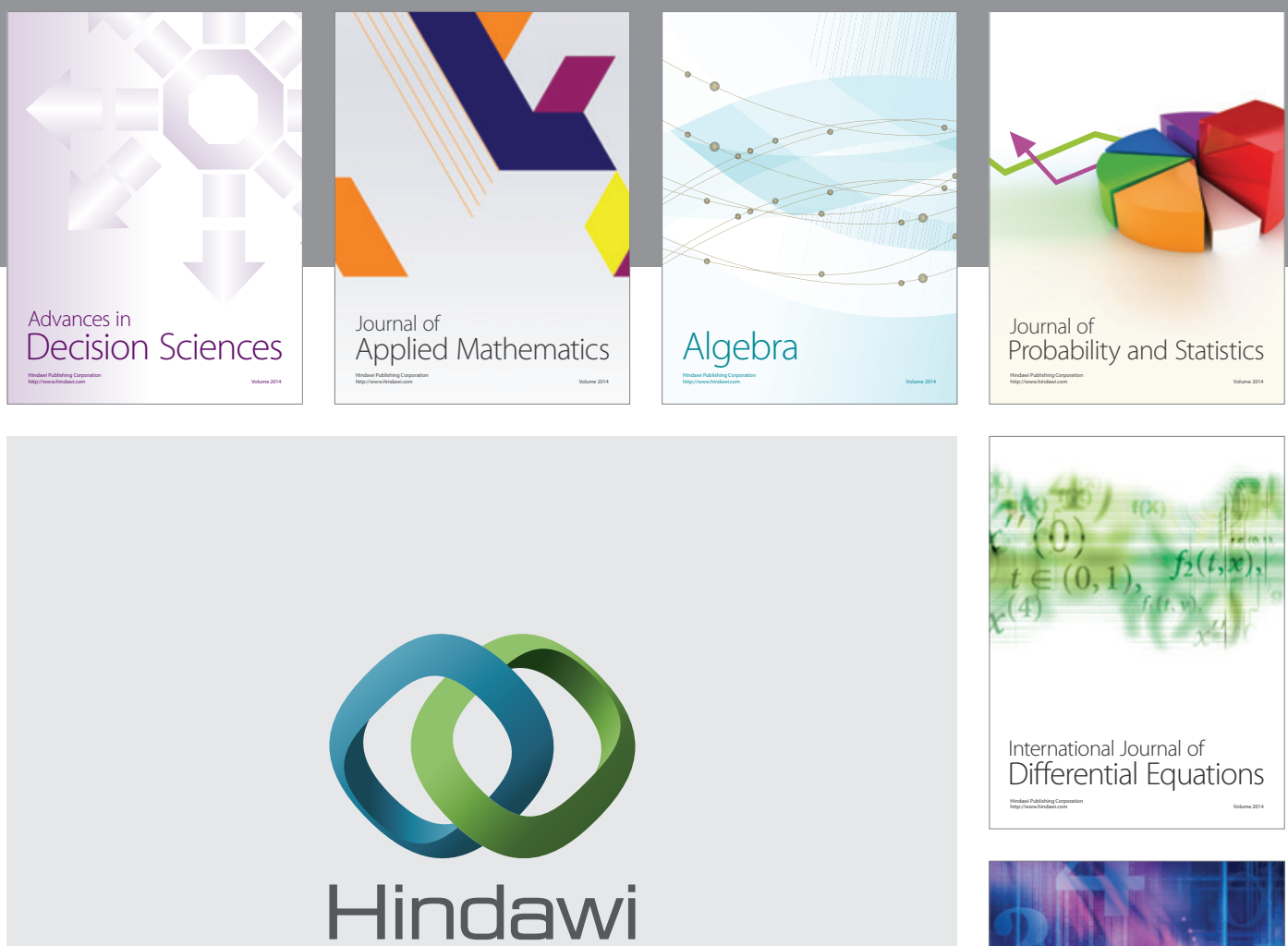

Submit your manuscripts at http://www.hindawi.com
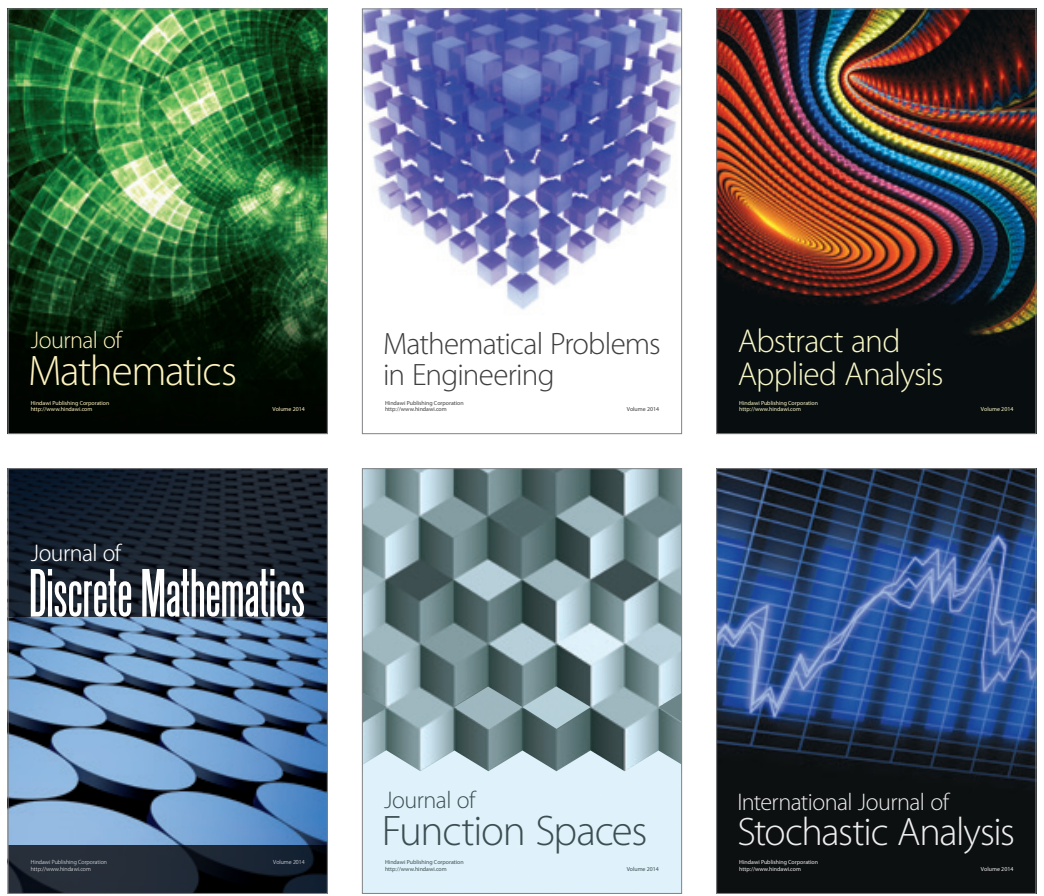

Journal of

Function Spaces

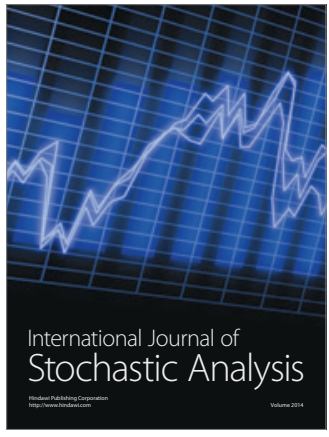

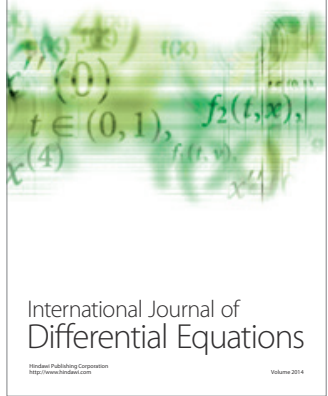
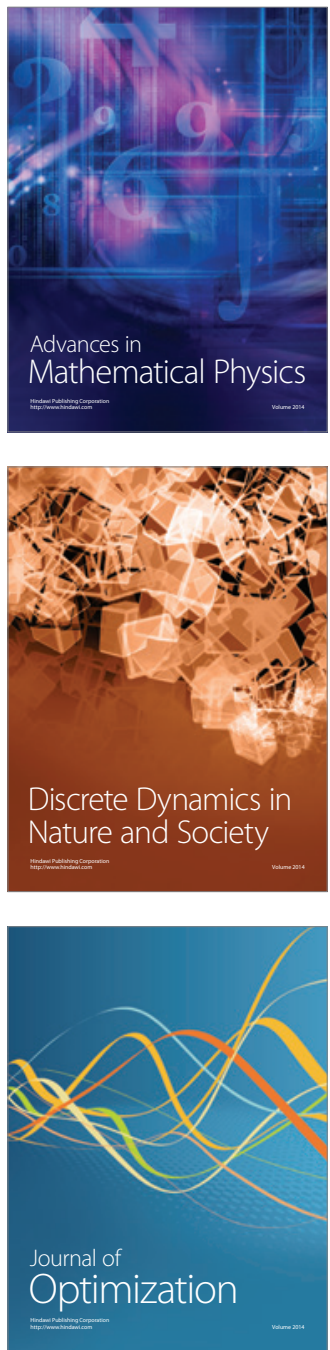